ACCEPTED BY APJ

Preprint typeset using LATEX style emulateapj v. 5/2/11

\title{
IDENTIFICATION OF OUTFLOWS AND CANDIDATE DUAL ACTIVE GALACTIC NUCLEI IN SDSS QUASARS AT $Z=0.8-1.6$
}

\author{
R. Scott Barrows ${ }^{1}$ Claud H. Sandberg Lacy, ${ }^{1,2}$ Julia Kennefick, ${ }^{1,2}$ Julia M. Comerford, ${ }^{3}$ Daniel Kennefick, ${ }^{1,2}$ \\ AND JOEL C. BERRIER ${ }^{1,2}$ \\ accepted by $A p J$
}

\begin{abstract}
We present a sample of 131 quasars from the Sloan Digital Sky Survey at redshifts $0.8<z<1.6$ with double peaks in either of the high-ionization narrow emission lines [Ne V] $\lambda 3426$ or [Ne III] $\lambda 3869$. These sources were selected with the intention of identifying high-redshift analogs of the $z<0.8$ active galactic nuclei (AGN) with double-peaked [O III] $\lambda 5007$ lines, which might represent AGN outflows or dual AGN. Lines of high-ionization potential are believed to originate in the inner, highly photoionized portion of the narrow line region (NLR), and we exploit this assumption to investigate the possible kinematic origins of the double-peaked lines. For comparison, we measure the $[\mathrm{Ne} \mathrm{V}] \lambda 3426$ and $[\mathrm{Ne} \mathrm{III}] \lambda 3869$ double peaks in low-redshift $(z<0.8)[\mathrm{O}$ III]-selected sources. We find that $[\mathrm{Ne} \mathrm{V}] \lambda 3426$ and [Ne III] $\lambda 3869$ show a correlation between line-splitting and line-width similar to that of [O III $] \lambda 5007$ in other studies; and the velocity-splittings are correlated with the quasar Eddington ratio. These results suggest an outflow origin for at least a subset of the double-peaks, allowing us to study the high-ionization gas kinematics around quasars. However, we find that a non-neligible fraction of our sample show no evidence for an ionization stratification. For these sources, the outflow scenario is less compelling, leaving the dual AGN scenario as a viable possibility. Finally, we find that our sample shows an anti-correlation between the velocity-offset ratio and luminosity ratio of the components, which is a potential dynamical argument for the presence of dual AGN. Therefore, this study serves as a first attempt at extending the selection of candidate dual AGN to higher redshifts.
\end{abstract}

Subject headings: galaxies: active - galaxies: nuclei - quasars: emission lines

\section{INTRODUCTION}

Mergers of gas-rich galaxies are likely to play a key role in the growth of supermassive black holes (SMBHs) through accretion, particularly in the triggering of quasar phases (Sanders et al. 1988; Treister et al. 2010). Quasars may also represent an important phase in the evolution of galaxies due to radiative feedback (Silk \& Rees 1998; Kauffmann \& Haehnelt 2000). This sequence of events is a potential scenario linking the evolution of galaxies and the growth of SMBHs (Di Matteo et al. 2005; Hopkins et al. 2005). Interestingly, both the merger and quasar phases can manifest themselves as active galactic nuclei (AGN) with doublepeaked narrow emission lines in their spectra. Specifically, during a galaxy merger, when the SMBHs are separated by $\sim 1 \mathrm{kpc}$ and are actively accreting as a dual AGN (Comerford et al. 2009b) they will each produce Doppler-shifted emission from their narrow line regions (NLRs), resulting in a double-peaked profile in the integrated spectrum. In the feedback scenario, radiation from a single quasar can drive bi-conical outflows of the NLR (Arribas et al. 1996; Veilleux et al. 2001; Crenshaw et al. 2010a), producing similar emission line profiles (Zheng et al. 1990; Fischer et al. 2011).

AGN whose spectra exhibit double-peaked narrow

\footnotetext{
${ }^{1}$ Arkansas Center for Space and Planetary Sciences, University of Arkansas, Fayetteville, AR 72701

author email: rbarrows@uark.edu

${ }_{2}^{2}$ Physics Department, University of Arkansas, Fayetteville, AR 72701

${ }^{3}$ Astronomy Department, University of Texas at Austin, Austin, TX 78712
}

emission lines (i.e. they are best fit by two components) represent a sub-class of the AGN population. Extended, offset and double-peaked line profiles have been observed in the narrow emission lines of AGN since the earliest studies of AGN NLRs (Heckman et al. 1981). These complex narrow line profiles are observed in both Type 1 and Type 2 AGN (Crenshaw et al. 2010b), and they are generally most pronounced in forbidden transition lines of high ionization potentials (I.P.s). In particular, such observations have often focused on the $5007 \AA$ transition line of $[\mathrm{O}$ III $](I . P .=35.15 \mathrm{eV})$ since it is a relatively intense emission line produced by the ionizing continuum of AGN and is accessible in optical spectra (see Veilleux 1991 and Whittle 1992 for examples). However, there is even variation among the high-ionization lines, with those of the highest ionization potentials, such as [Ne III] $(I . P .=41.07 \mathrm{eV})$, and $[\mathrm{Ne} \mathrm{V}]($ I.P. $=97.16 \mathrm{eV})$, displaying the largest velocity offsets (De Robertis \& Osterbrock 1984; Sturm et al. 2002; Spoon \& Holt 2009).

In the dual AGN scenario, the two emission line peaks are produced by the orbital motion of two AGN within a single merger remnant galaxy. This interpretation is intriguing since $\mathrm{SMBH}$ reside in the bulges of galaxies, and dual SMBHs (kpc-scale separations) are a stage of galaxy mergers before the SMBHs coalesce. The existence of dual AGN has been confirmed observationally in several serendipitous cases, most notably in ultra-luminous infrared galaxies as pairs of X-ray point sources (Komossa et al. 2003; Guainazzi et al. 2005; Hudson et al. 2006; Bianchi et al.|2008; Piconcelli et al. 2010; Koss et al. 2011; Mazzarella et al. 2012). Sys- 
tematic searches for dual AGN in large spectroscopic databases, such as the Sloan Digital Sky Survey (SDSS; Abazajian et al. 2009) and DEEP2 (Newman et al. 2012) have involved identifying AGN with double emission line systems (Comerford et al. 2009b; Wang et al. 2009; Liu et al. 2010b; Smith et al. 2010; Ge et al. 2012). Promising results are being obtained through follow-up observations in the form of high-resolution optical imaging (Comerford et al. 2009a), near-infrared (NIR) adaptive optics imaging (Liu et al. 2010a; Fu et al. 2011a; Rosario et al. 2011; Shen et al. 2011a; Barrows et al. 2012), spatially resolved spectroscopy (McGurk et al. 2011; Fu et al. 2012), hard X-ray observations (Comerford et al. 2011; Civano et al. 2012; Liu et al. 2012a), radio observations (Fu et al. 2011b), and diagnostics deduced statistically from longslit spectroscopy (Comerford et al. 2012). A sample of dual AGN will be useful in studying the connection between galaxy interactions/mergers and AGN activity (Green et al. 2010; Liu et al. 2011, 2012b), and for refining the galaxy merger rate (Conselice et al.|2003; Berrier et al. 2006; Lotz et al. 2011; Berrier \& Cooke 2012).

In the outflow scenario, offset or double-peaked narrow emission lines are often attributed to radially flowing NLR gas driven by energy from the AGN. Furthermore, this effect is also thought to produce a stratification of the NLR since the incident ionizing flux and electron density should diminish with increasing distance from the nuclear source. This will result in the production of different lines in varying proportions as a function of radius from the AGN (Veilleux 1991). Observationally, luminous quasars are known to have extended NLRs driven by energy from the AGN coupled to the interstellar medium gas (Bennert et al. 2002), and powerful radio galaxies often show complex, spatially extended NLRs with multiple components aligned along the radio axis. Models for this alignment include reflection of the AGN emission (Tadhunter et al. 1988) or material entrained in a radio jet (Holt et al. 2003, 2008; Komossa et al.|2008). In AGN with sufficiently high Eddington ratios, radiation pressure acting on gas and dust (Everett 2007) or a hot wind that entrains the NLR clouds (Everett \& Murray 2007) are possible mechanisms capable of driving the line splitting. Whichever mechanism is the dominant driver of outflows in an individual source, they may represent cases of AGN feedback, which might be important in quenching star formation in galaxies following a merger and in establishing the observed correlations between SMBH masses and host galaxy properties (Ferrarese \& Merritt 2000; Marconi \& Hunt 2003).

Whether AGN with double-peaked emission lines represent dual AGN or powerful AGN outflows, they have proven useful for investigating several aspects of AGN evolution, including AGN triggering and feedback. Systematic searches for double-peaked AGN have primarily utilized the $[\mathrm{O}$ III $] \lambda 5007$ emission line and have therefore been limited to below redshifts of $z \approx 0.80$ since $[\mathrm{O}$ III $] \lambda 5007$ is not accessible in optical spectra at higher redshifts. However, there is evidence that at high redshifts galaxy mergers were more prevalent, and AGN outflows might have played an important role in the evolution of galaxies at $z \geq 1$, including massive radio galaxies (Nesvadba et al. 2008). Furthermore, there is significant controversy over the role that galaxy mergers play in AGN activity and SMBH growth at high redshifts (Cisternas et al. 2011; Treister et al. 2012; Kocevski et al. 2012). Therefore, a sample of high redshift double-peaked narrow line AGN will be important for investigating these questions. So far only one such candidate dual AGN has been identified above $z \sim 0.8$ as a serendipitous discovery at $z=1.175$ through doublepeaked UV and optical emission lines, particularly evident in $[\mathrm{Ne} \mathrm{V}] \lambda 3426$ and [Ne III] $\lambda 3869$ (Barrows et al. 2012). Motivated by this discovery, we have conducted a systematic search for additional AGN at high redshift $(z>0.8)$ with double-peaked [Ne V] 23426 and [Ne III] $\lambda 3869$ emission lines. These emission lines have relatively high ionization potentials and trace the gas photoionized by the AGN continuum. Furthermore, they are likely to originate in the inner (more highly ionized) portion of the NLR, allowing for the identification of AGN driven outflows at $0.8<z<1.6$. This study will also serve as a first attempt at extending the identification of candidate dual AGN to higher redshifts.

In Section 2 we describe our parent sample and how we selected our final sample and low-redshift comparison sample. In Section 3 we describe the systemic redshifts we will use throughout the paper. In Section 4 we describe the general properties of our sample and our estimates of selection completeness. In Section 5 we investigate several correlations among the emission line properties that aid in illuminating the origin of the double-peaked line profiles. In Section [6] we describe the radio properties of our sample and compare them to our parent sample. In Section 7 we discuss the most likely physical mechanisms driving the line-splitting, particularly focusing on the scenarios of AGN outflows and dual AGN. In Section 8 we summarize our main conclusions. Throughout the paper we adopt the cosmological parameters $\Omega_{\Lambda}=0.728, \Omega_{b}=0.0455, \Omega_{m} h^{2}=0.1347$, and $H_{0}=70.4 \mathrm{~km} \mathrm{~s}^{\text {?1 }} \mathrm{Mpc}^{\text {?1 }}$. This corresponds to the maximum likelihood cosmology from the combined $\mathrm{WMAP}+\mathrm{BAO}+\mathrm{H} 0$ results from the WMAP 7 data release of Komatsu et al. (2011).

\section{GENERATING THE SAMPLE}

\subsection{Parent Sample}

Our parent sample consists of archival spectra drawn from the quasar catalog of the SDSS Data Release 7 (DR7) which is described in detail in Schneider et al. $(2010)$. The typical resolution of the SDSS spectra is $\lambda / \Delta \lambda \sim 2000$. In short, inclusion in the catalog requires luminosities brighter than $M_{i}=-22.0$, at least one emission line with FWHM $>1000 \mathrm{~km} \mathrm{~s}^{-1}$ or complex absorption features, apparent magnitudes fainter than $i \approx 15.0$, and have highly reliable redshifts (see Section 3 for a discussion of the redshifts). We restricted the lower redshift limit of the sample to $z \geq 0.80$ to only include sources not in the parent samples of [O III]-selected double-peaked emitters from the SDSS (Wang et al. 2009; Liu et al. 2010b; Smith et al. 2010; Ge et al. 2012) since our intention is to select sources which are not identifiable by their methods. Additionally, we required that at least $[\mathrm{Ne} \mathrm{V}] \lambda 3426$ be accessible in the SDSS wavelength range $(3800-9200 \AA)$, which imposes an upper limit of $z \sim 1.7$. We did not make any selection 
cuts based on the signal-to-noise ratios $(\mathrm{S} / \mathrm{N})$. This resulted in a parent sample of 39,876 sources.

\subsection{Initial Selection}

Our initial selection involved visually identifying quasars from the parent sample (Section 2.1) with detectable double emission line peaks in $[\mathrm{Ne} \mathrm{V}] \lambda 3426$ and/or $[\mathrm{Ne}$ III $] \lambda 3869$. These two lines were used since they are accessible in the SDSS optical wavelength range at $z>0.80$, are relatively strong narrow lines in quasar spectra, and are not severely blended with any other strong lines. We did not require that two explicit peaks be detectable in both of those emission lines for two reasons: 1) the ionizing continuum may be such that $[\mathrm{Ne} \mathrm{V}] \lambda 3426$ is too weak to be detected whereas [Ne III] $\lambda 3869$ is detectable; and 2) a difference in line ratios and/or velocity-splittings between the two lines may result in one pair being more blended than the other. Therefore, since the purpose of this analysis is to investigate the origin of the double-peaked emission lines, we did not want to exclude those sources which show variations among the line properties. While $[\mathrm{O}$ II $] \lambda 3727$ is another strong emission line accessible in most of our parent sample, we did not select sources based on this line since $[\mathrm{O}$ II $] \lambda 3727$ is a doublet $(\lambda 3726,3729 \AA)$ with $\sim 200 \mathrm{~km} \mathrm{~s}^{-1}$ separation between the transition wavelengths and is difficult to discern from true peaks, particularly with the limited spectral resolution of the SDSS (see Smith et al. 2010 for a similar discussion). In sources where the double peak detections are ambiguous in each line, corresponding peaks in both $[\mathrm{Ne} \mathrm{V}] \lambda 3426$ and [Ne III] $\lambda 3869$ are needed for confirmation. Out of the parent sample, our visual selection process resulted in 181 preliminary sources.

\subsection{Modeling the Spectra and Selection of the Final Sample}

To generate the final sample to be used in our subsequent analyses, we modeled each spectrum yielded by the initial selection stage (Section 2.2) in order to determine if a two-Gaussian model significantly improves the line profile fit over a single-Gaussian model. This modeling proceeded in two stages: 1) continuum modeling, and 2) emission line modeling.

\subsubsection{Continuum Modeling}

The continuum was modeled by masking all detectable emission lines and simultaneously fitting a power-law function $\left(F_{\lambda} \sim \lambda^{-\alpha_{\lambda}}\right)$ for the underlying AGN contiuum radiation plus a pseudo-continuum of broadened Fe II emission lines from the empirical templates of Tsuzuki et al. (2006) $\left(\lambda_{\text {obs }}<3500 \AA\right)$ and Véron-Cetty et al. (2004) $\left(\lambda_{\text {obs }}>3500 \AA\right)$ which were developed from the spectrum of the narrow-line Seyfert 1 galaxy I Zw 1. Due to the use of two separate Fe II templates, the powerlaw function was allowed to have a break at $3500 \AA$, and the Fe II normalization allowed to vary independently below and above the break wavelength. The Fe II pseudo-continuum is composed of many blended Fe II transitions which are generally believed to originate in or near the classical broad line re- gion (BLR), and as such the redshift and broadening of the Fe II emission should be comparable to that of the broad emission lines. Therefore, in our modeling the Fe II emission was fixed at the redshift of the quasar being modeled (see Section 3 for a detailed discussion of the redshifts); we broadened the Fe II template by convolving with a Gaussian of $F W H M_{\text {conv }}$ where $F W H M_{\mathrm{FeII}}^{2}=F W H M_{\mathrm{conv}}^{2}+F W H M_{\mathrm{I} \mathrm{Zw} 1}^{2}$ and $F W H M_{\mathrm{I} \mathrm{Zw} 1}=900 \mathrm{~km} \mathrm{~s}^{-1}$. Use of this template necessarily limits the minimum $F W H M_{F e I I}$ best-fit to $900 \mathrm{~km} \mathrm{~s}^{-1}$, though this was not a problem since these sources were in the quasar catalog based on the presence of broad lines with $F W H M>1000 \mathrm{~km} \mathrm{~s}^{-1}$. The lower rest-wavelength end of the continuum+Fe II fitting window was $2750 \AA$ and the upper rest-wavelength end was $3950 \AA$ (just redward of [Ne III $] \lambda 3869$ ) if accessible, or otherwise the red end of the spectral coverage. This fitting window allowed proper detection of the Fe II emission since it has a relatively large equivalent width near $\mathrm{Mg}$ II $\lambda 2800$. We allowed $F W H M_{\mathrm{FeII}}$ to vary in steps of $100 \mathrm{~km} \mathrm{~s}^{-1}$, and in general, the solutions are in the range $\sim 2000-9000 \mathrm{~km} \mathrm{~s}^{-1}$, though in most cases the quality of the fits were not strongly dependent on the broadening.

\subsubsection{Narrow Emission Line Modeling}

We fit each emission line ([Ne $\mathrm{V}] \lambda 3426$ and [Ne III] $\lambda 3869)$ with both a single and a double Gaussian model, and required that sources in our final sample have fits to at least one of these two lines significantly improved by the double Gaussian model $\left(\Delta \chi^{2} \geq\right.$ $\left.\Delta n_{D O F}\right)$. In seven individual line models, an additional broad component of line width FWHM $\approx$ $700-1500 \mathrm{~km} \mathrm{~s}^{-1}$ was required for a satisfactory fit. Three of the $[\mathrm{Ne} \mathrm{V}] \lambda 3426$ fits required such a component, where in two of those cases the broad component is consistent with the blue narrow peak (J074242.18+374402.0: $F W H M=1470 \mathrm{~km} \mathrm{~s}^{-1}$; and J105035.57+190544.2: $F W H M=1220 \mathrm{~km} \mathrm{~s}^{-1}$ ), and in the third case it is consistent with the red narrow peak (J150243.93+281739.9: $\left.F W H M=1320 \mathrm{~km} \mathrm{~s}^{-1}\right)$. Four of the $[\mathrm{Ne}$ III $] \lambda 3869$ fits required a broad component, where in three of those cases it is consistent with the blue narrow peak (J105035.57+190544.2: $F W H M=1520$ $\mathrm{km} \mathrm{s}^{-1}$; J105634.56+121023.5: $F W H M=820 \mathrm{~km} \mathrm{~s}^{-1}$; and J145659.27+503805.4: $\left.F W H M=700 \mathrm{~km} \mathrm{~s}^{-1}\right)$ and in one those cases it is located between the blue and red peaks (J085205.91+183922.2: $F W H M=1020$ $\left.\mathrm{km} \mathrm{s}^{-1}\right)$. We note that in only one of these sources $(\mathrm{J} 105035.57+190544.2)$ is a broad component seen in both $[\mathrm{Ne} \mathrm{V}] \lambda 3426$ and $[\mathrm{Ne} \mathrm{III]} \lambda 3869$, and it is consistent with the blue peak.

Properly accounting for the contribution of flux from Fe II emission is often important in determining the profiles of the emission lines of interest in this wavelength range. In particular, there is a local peak in the broadened Fe II emission just blueward of [Ne V] $\lambda 3426$, between $3390 \AA$ and $3410 \AA$, which has the potential to complicate the isolation of the blue $[\mathrm{Ne} \mathrm{V}] \lambda 3426$ component. Therefore, careful attention was paid to the modeling in this region, and any sources for which the model was ambiguous were not admitted into the final sample. However, there is no significant Fe II emission near $[\mathrm{Ne}$ III $] \lambda 3869$, and only a small local peak redward of 

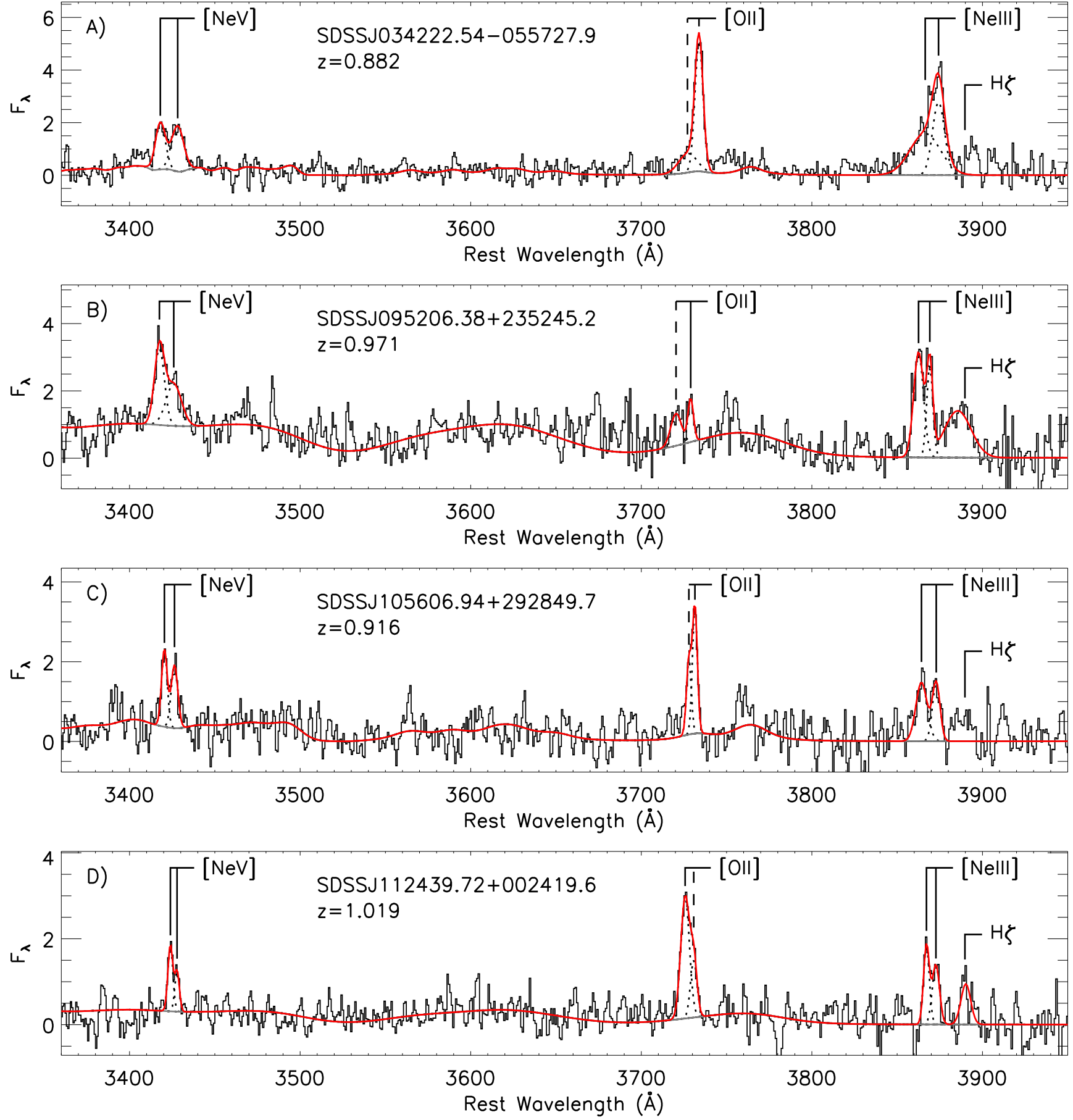

FIG. 1. - Four examples of quasar spectra yielded by our selection process shown in the quasar rest-frame $\left(z_{S D S S}\right)$ wavelength range of $3360-3950 \AA$. The spectra have been smoothed by convolving with a Gaussian of $\sigma=1 \AA$. The flux densities, $F_{\lambda}$, are in units of $10^{-17}$ $\mathrm{erg} \mathrm{s}^{-1} \mathrm{~cm}^{-2} \AA^{-1}$. In each of the four panels the power-law continuum has been subtracted from the spectrum, and the Fe II template (gray, solid line), best-fit Gaussians (black, dotted lines), and the best-fit model sum (red, solid line) are shown. See Section 2.3 for details on fitting the models. These are examples for which double components are measurable in both [Ne V] $\lambda 3426$ and [Ne III] $\lambda 3869$. In all four cases we can only place upper limits on the presence of a second [O II] $] 3727$ component (vertical dashed line). In each panel we have labeled the expected position of $\mathrm{H} \zeta$ based on its rest-wavelength, however it is not always detected, and in no cases can we resolve double-peaks in $\mathrm{H} \zeta$. 

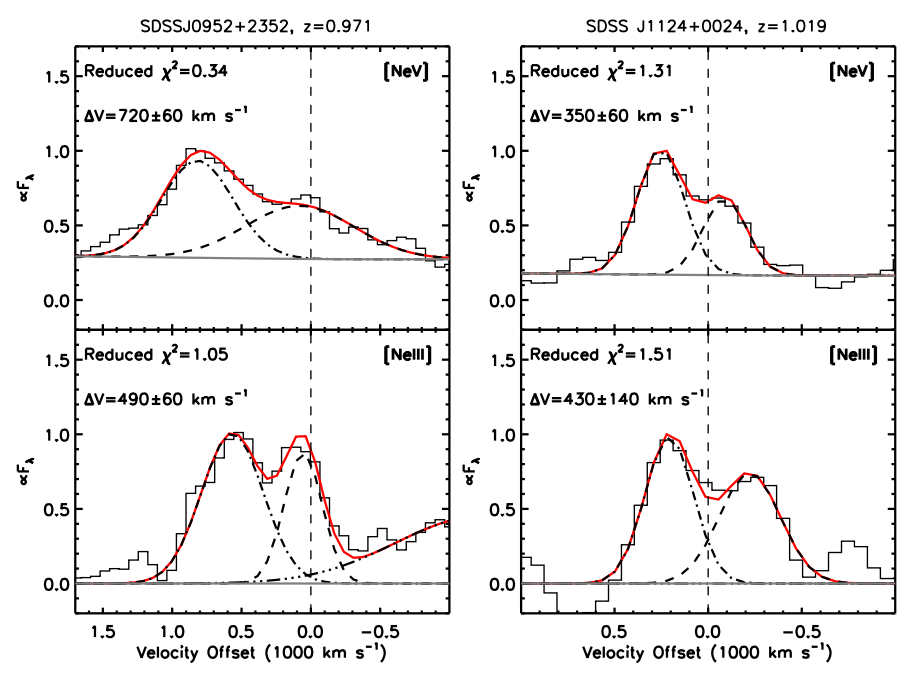

FIG. 2. - Examples of two spectra from Figure 1 zoomed-in on $[\mathrm{Ne} \mathrm{V}] \lambda 3426$ (top) and [Ne III] $\lambda 3869$ (bottom). The spectra have been smoothed by convolving with a Gaussian of $\sigma=2 \AA$. In each panel the peak flux of the double Gaussian model has been normalized to unity, and the spectra are plotted in velocity-space with zero-velocity at the quasar redshift. The power-law continuum has been subtracted from the spectrum, and the model components are the same as described in Figure 1

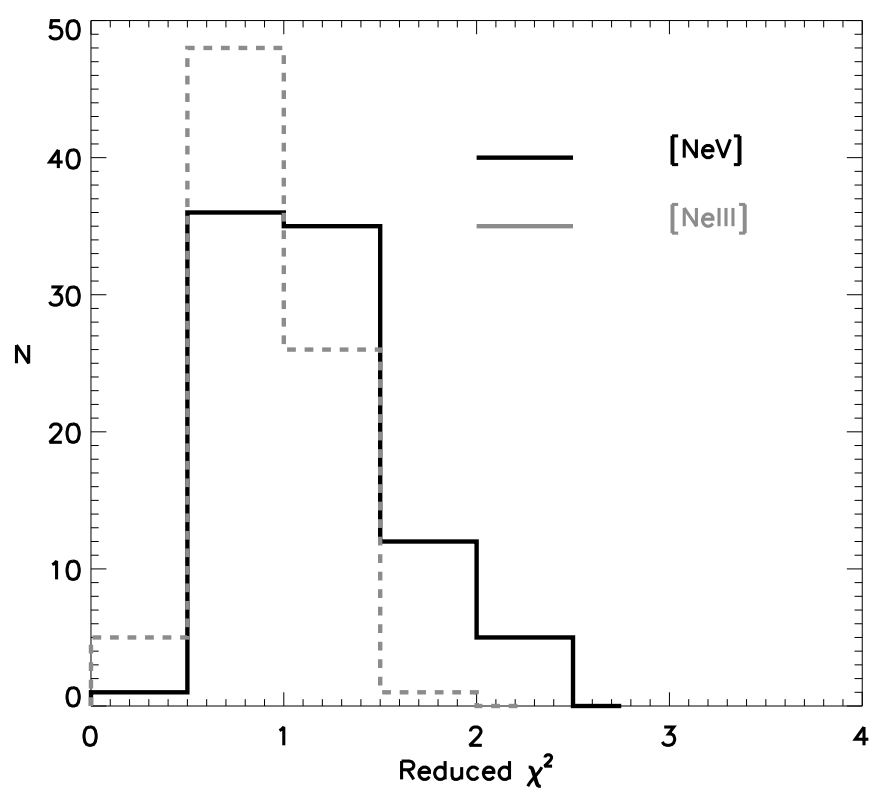

FIG. 3. - Distribution of reduced $\chi^{2}$ values for the best double-Gaussian fits for [Ne V] $\lambda 3426$ (black, solid line) and [Ne III] $\lambda 3869$ (gray, dashed line).

[O II] $] 33727$ but with which it is not blended.

From our spectral modeling, there are 38 sources in our sample for which we have measured robust doublepeaks in both $[\mathrm{Ne} \mathrm{V}] \lambda 3426$ and [Ne III] $\lambda 3869,42$ sources for which we have only robust [Ne V] $\lambda 3426$ measurements, and 51 sources for which we have only robust [Ne III] $\lambda 3869$ measurements. Thus, there are a total of 131 double-peaked sources in our sample, making the fraction of double-peaked AGN detected in this manner $\sim 0.3 \%$ of the parent sample. See Figure 1 for examples of the spectra in our final sample show- ing the best-fit models over the rest-wavelength range containing $[\mathrm{Ne} \mathrm{V}] \lambda 3426$ and $[\mathrm{Ne} \mathrm{III]} \lambda 3869$. Additionally, Figure 2 compares the individual $[\mathrm{Ne} \mathrm{V}] \lambda 3426$ and [Ne III] $\lambda 3869$ line profiles in velocity-space. The best-fit parameters of the emission line modeling are listed in Table 1 ([Ne V] $\lambda 3426)$ and Table $2([\mathrm{Ne}$ III $] \lambda 3869)$. All errors correspond to $1 \sigma$ uncertainties and have been propagated throughout any further calculations. The reduced $\chi^{2}$ values for each double Gaussian model are listed in Tables 1 and 2 and shown as a distribution in Figure 3 , The emission line modeling was performed with SPECFIT (Kriss 1994). Our best-fitting Fe II FWHMs are listed in Table 3 , along with other relevant quasar properties (redshifts, Mg II $\lambda 2800$ FWHMs, and Eddington ratios) which will be described in subsequent sections.

Throughout the rest of the paper, the velocity offsets of the individual blue and red components will refer to the offsets from the systemic velocity. For $[\mathrm{Ne} \mathrm{V}] \lambda 3426$, these blue and red velocity offsets will be defined as $\Delta V_{[\mathrm{NeV}] \text {, blue }}$ and $\Delta V_{[\mathrm{NeV}] \text {,red }}$, respectively. For $[\mathrm{Ne}$ III $] \lambda 3869$, the blue and red velocity offsets will be defined as $\Delta V_{[\mathrm{NeIII}] \text {,blue }}$ and $\Delta V_{[\mathrm{NeIII}] \text {,red }}$, respectively. Blueshifts will correspond to positive velocities. The velocity-splittings will then be the velocity difference between the lines, i.e. $\Delta V_{\mathrm{NeV}} \equiv \Delta V_{[\mathrm{NeV}] \text {,blue }}-\Delta V_{[\mathrm{NeV}] \text {,red }}$ and $\Delta V_{\text {NeIII }} \equiv \Delta V_{\text {[NeIII],blue }}-\Delta V_{\text {[NeIII],red }}$. The redshifts corresponding to the systemic velocity of each source are described in Section 3 .

\subsection{Comparison Sample}

To generate a $z<0.8$ comparison sample to be used in our analyses, we examined the $89[\mathrm{O}$ III]-selected Type 1 sources in Smith et al. (2010) and measured the line properties of double-peaked [Ne V] $\lambda 3426$ and [Ne III] $\lambda 3869$ in cases for which two peaks are detectable in those lines. We chose this sample because, as opposed to other double-peaked [O III] $\lambda 5007$ samples, it includes Type 1 AGN which were selected from the SDSS quasar catalog. Therefore, it provides a $z<0.8$ sample which can be used for direct comparison. For the measurements on the $[\mathrm{O} \mathrm{III}]$-selected sample, we selected those with robust $[\mathrm{Ne} \mathrm{V}] /[\mathrm{Ne} \mathrm{III}]$ double-peaks in the same way as for our high-redshift sample, and likewise modeled the spectra as described above. This resulted in 18 sources in the [O III]-selected comparison sample for which we have measured robust double-peaks in both $[\mathrm{Ne} \mathrm{V}] \lambda 3426$ and [Ne III] $\lambda 3869,4$ sources for which we have only have robust [Ne V] $\lambda 3426$ measurements, and 20 sources for which we have only have robust [Ne III] $\lambda 3869$ measurements. Thus, there are a total of 42 double-peaked sources in our comparison sample.

\section{REDSHIFTS}

Some sections of our analysis require a knowledge of the individual quasar redshifts. For the sources in our sample, we have redshifts available from several different measurement techniques. In this section we describe those redshift estimates which are useful for our scientific interests.

\subsection{SDSS Redshifts}

Redshifts for spectroscopically-detected sources in the SDSS are determined by the 'spectro1d' code described 
TABLE 1

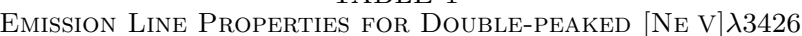

\begin{tabular}{|c|c|c|c|c|c|c|c|}
\hline \multicolumn{4}{|c|}{ Blue } & \multicolumn{3}{|c|}{ Red } & \multirow[b]{2}{*}{ Reduced $\chi^{2}$ (dof) } \\
\hline SDSS Name & $\mathrm{F}_{\lambda}^{\mathrm{a}}$ & $\Delta V^{\mathrm{b}}$ & $\mathrm{FWHM}^{\mathrm{c}}$ & $\overline{\mathrm{F}_{\lambda}{ }^{\mathrm{a}}}$ & $\Delta V^{\mathrm{b}}$ & $\mathrm{FWHM}^{\mathrm{c}}$ & \\
\hline J003159.87+063518.8 & $5.4 \pm 4$ & $334 \pm 124$ & $457 \pm 268$ & $6.9 \pm 4$ & $-128 \pm 6$ & $465 \pm 203$ & $0.58(10)$ \\
\hline J014933.86+143142.6 & $4.7 \pm 2$ & $959 \pm 55$ & $260 \pm 97$ & $18.7 \pm 4$ & $319 \pm 57$ & $731 \pm 209$ & $1.25(42)$ \\
\hline J015325.74+145233.4 & $28.3 \pm 8$ & $504 \pm 49$ & $403 \pm 119$ & $33.9 \pm 11$ & $-176 \pm 68$ & $585 \pm 249$ & $0.70(32)$ \\
\hline J021648.36-092534.3 & $7.0 \pm 2$ & $930 \pm 35$ & $245 \pm 50$ & $34.9 \pm 5$ & $184 \pm 41$ & $802 \pm 131$ & $0.65(43)$ \\
\hline J021703.10-091031.1 & $16.4 \pm 4$ & $871 \pm 35$ & $364 \pm 86$ & $28.0 \pm 5$ & $155 \pm 51$ & $671 \pm 156$ & $0.82(30)$ \\
\hline J034222.54-055727.9 & $23.8 \pm 6$ & $746 \pm 73$ & $641 \pm 206$ & $24.4 \pm 6$ & $-136 \pm 83$ & $682 \pm 176$ & $0.90(85)$ \\
\hline J035230.55-071102.3 & $149.1 \pm 100$ & $690 \pm 270$ & $933 \pm 267$ & $244.1 \pm 108$ & $-15 \pm 110$ & $788 \pm 185$ & $0.72(79)$ \\
\hline J073408.62+411901.1 & $10.8 \pm 4$ & $589 \pm 81$ & $364 \pm 214$ & $20.1 \pm 5$ & $-11 \pm 68$ & $469 \pm 151$ & $1.95(35)$ \\
\hline J074242.18+374402.0* & $9.9 \pm 5$ & $198 \pm 34$ & $236 \pm 125$ & $10.3 \pm 4$ & $-116 \pm 30$ & $209 \pm 74$ & $0.81(33)$ \\
\hline J074641.70+352645.6 & $38.9 \pm 22$ & $1070 \pm 327$ & $1306 \pm 476$ & $22.8 \pm 21$ & $124 \pm 254$ & $961 \pm 373$ & $0.84(58)$ \\
\hline
\end{tabular}

Note. - All errors correspond to $1 \sigma$ uncertainties. ${ }^{*}$ denotes that a broad component was necessary. This table is available in its entirety in a machine-readable form in the online journal. A portion is shown here for guidance regarding its form and content.

${ }^{a}$ Flux densities, $F_{\lambda}$, are in units of $10^{-17} \mathrm{erg} \mathrm{s}^{-1} \mathrm{~cm}^{-2} \AA^{-1}$.

b $\Delta V$ is velocity offset from the quasar redshift in $\mathrm{km} \mathrm{s}^{-1}$.

${ }^{c}$ FWHM are the observed line widths in units of $\mathrm{km} \mathrm{s}^{-1}$.

TABLE 2

Emission Line Properties for Double-Peaked [Ne III] $\lambda 3869$

\begin{tabular}{|c|c|c|c|c|c|c|c|}
\hline & & Blue & & & Red & & \\
\hline SDSS Name & $\mathrm{F}_{\lambda}{ }^{\mathrm{a}}$ & $\Delta V^{\mathrm{b}}$ & FWHM $^{\mathrm{c}}$ & $\mathrm{F}_{\lambda}^{\mathrm{a}}$ & $\Delta V^{\mathrm{b}}$ & FWHM $^{\mathrm{c}}$ & Reduced $\chi^{2}$ (dof) \\
\hline J000531.41+001455.9 & $28.0 \pm 7$ & $146 \pm 53$ & $372 \pm 125$ & $11.2 \pm 5$ & $-179 \pm 32$ & $125 \pm 49$ & $2.15(30)$ \\
\hline J003159.87+063518.8 & $6.2 \pm 11$ & $446 \pm 139$ & $223 \pm 278$ & $18.1 \pm 12$ & $61 \pm 232$ & $560 \pm 274$ & $2.07(31)$ \\
\hline J004305.92-004637.6 & $7.8 \pm 6$ & $395 \pm 74$ & $236 \pm 276$ & $9.5 \pm 6$ & $111 \pm 55$ & $209 \pm 151$ & $1.20(32)$ \\
\hline J004312.70+005605.0 & $9.3 \pm 2$ & $215 \pm 31$ & $215 \pm 65$ & $16.6 \pm 2$ & $-140 \pm 13$ & $212 \pm 24$ & $1.32(33)$ \\
\hline J014822.62+132142.7 & $37.8 \pm 15$ & $883 \pm 78$ & $1048 \pm 438$ & $27.6 \pm 6$ & $-376 \pm 102$ & $779 \pm 149$ & $0.83(47)$ \\
\hline J015734.24+003405.5 & $17.1 \pm 5$ & $806 \pm 50$ & $260 \pm 93$ & $70.6 \pm 12$ & $-69 \pm 42$ & $672 \pm 131$ & $1.05(37)$ \\
\hline J021648.36-092534.3 & $8.9 \pm 2$ & $538 \pm 27$ & $185 \pm 50$ & $35.8 \pm 5$ & $-4 \pm 36$ & $546 \pm 95$ & $1.06(24)$ \\
\hline J023234.33-091053.0 & $51.4 \pm 17$ & $1462 \pm 157$ & $1565 \pm 506$ & $34.4 \pm 10$ & $31 \pm 151$ & $1121 \pm 238$ & $0.73(66)$ \\
\hline J034222.54-055727.9 & $28.8 \pm 9$ & $480 \pm 0$ & $925 \pm 302$ & $58.4 \pm 7$ & $-323 \pm 37$ & $656 \pm 81$ & $1.09(78)$ \\
\hline J035230.55-071102.3 & $120.0 \pm 42$ & $382 \pm 68$ & $433 \pm 135$ & $201.2 \pm 40$ & $-70 \pm 33$ & $379 \pm 57$ & $0.95(32)$ \\
\hline
\end{tabular}

Note. - All labels are the same as in Table 1] This table is available in its entirety in a machine-readable form in the online journal. A portion is shown here for guidance regarding its form and content.

TABLE 3

General Quasar Properties for Our Sample

\begin{tabular}{cccccc}
\hline \hline SDSS Name & $\mathrm{z}_{\text {SDSS }}$ & $\mathrm{z}_{\mathrm{MgII}}$ & $\mathrm{FWHM}_{\text {FeII }}{ }^{\mathrm{a}}$ & $\mathrm{FWHM}_{\mathrm{MGII}}{ }^{\mathrm{a}, \mathrm{b}}$ & $\mathrm{f}_{\mathrm{Edd}}{ }^{\mathrm{c}}$ \\
\hline J000531.41+001455.9 & $0.9918 \pm 0.0009$ & $0.9931 \pm 0.0018$ & 9000 & $5424.76 \pm 1192.59$ & 0.109 \\
J003159.87+063518.8 & $1.0921 \pm 0.0010$ & $1.0935 \pm 0.0016$ & 1000 & $2475.19 \pm 1745.19$ & 0.085 \\
J004305.92-004637.6 & $0.8482 \pm 0.0015$ & $0.8488 \pm 0.0015$ & 8400 & $2828.74 \pm 247.77$ & 0.094 \\
J004312.70+005605.0 & $0.9036 \pm 0.0010$ & $0.9047 \pm 0.0014$ & 8500 & $6223.22 \pm 283.39$ & 0.105 \\
J014822.62+132142.7 & $0.8767 \pm 0.0018$ & $0.8759 \pm 0.0019$ & 8000 & $6063.22 \pm 134.05$ & 0.146 \\
J014933.86+143142.6 & $0.9024 \pm 0.0013$ & $0.9027 \pm 0.0013$ & 4500 & $2969.71 \pm 244.58$ & 0.172 \\
J015325.74+145233.4 & $1.1755 \pm 0.0020$ & $1.1762 \pm 0.0021$ & 5100 & $5219.56 \pm 553.29$ & 0.202 \\
J015734.24+003405.5 & $1.0834 \pm 0.0013$ & $1.0825 \pm 0.0020$ & 3300 & $6846.37 \pm 1636.32$ & 0.109 \\
J021648.36-092534.3 & $0.8834 \pm 0.0013$ & $0.8834 \pm 0.0014$ & 2700 & $4288.12 \pm 632.18$ & 0.119 \\
J021703.10-091031.1 & $0.8752 \pm 0.0013$ & $0.8757 \pm 0.0015$ & 2700 & $2598.31 \pm 432.35$ & 0.289 \\
\hline
\end{tabular}

Note. - This table is available in its entirety in a machine-readable form in the online journal. A portion is shown here for guidance regarding its form and content.

${ }^{\text {a }}$ FWHMs are in units of $\mathrm{km} \mathrm{s}^{-1}$.

b Values for FWHM $M_{M I I}$ are from the SDSS DR7 Catalog of Quasar Properties (Shen et al. 2011b).

${ }^{\mathrm{c}}$ Eddington ratio calculations are described in Section [5.2 


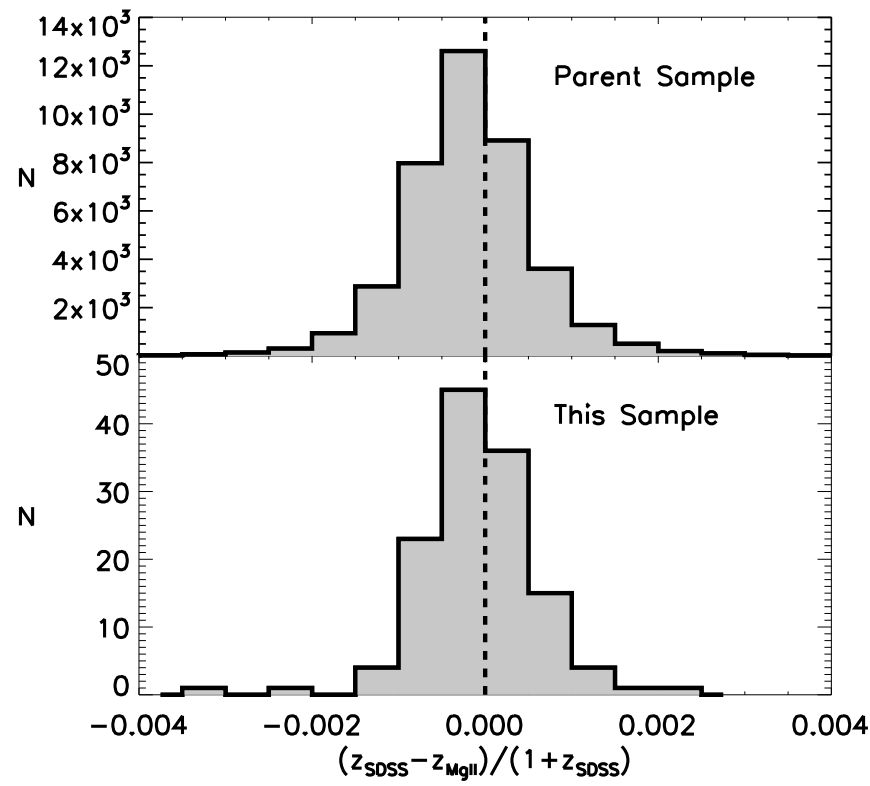

FIG. 4.- Distribution of $\left(z_{S D S S}-z_{M g I I}\right) /\left(1+z_{S D S S}\right)$ for the parent sample (top) and our sample (bottom). The mean and sigma are shown for each distribution. Note that the distributions are similar for both samples.

in Stoughton et al. (2002). In short, two separate redshifts are determined, an emission line redshift $\left(z_{E L}\right)$ and a cross-correlation redshift $\left(z_{X C}\right)$, and the final value adopted by the code is the redshift solution with the highest confidence level (though each individual value is stored and available through the archive). Additionally, a small fraction of the quasar redshifts were re-determined after visual inspection as described in Schneider et al. (2010). $z_{E L}$ is measured by the identification of common galaxy and quasar emission lines with known rest-wavelength values, and therefore for our sample the highest confidence $z_{E L}$ values are almost exclusively determined by sets of the strong quasar emission lines C IV $\lambda 1549, \mathrm{C}$ III $\lambda 1$ 1909, Mg III $\lambda 2800,[\mathrm{O}$ II $] \lambda 3727$, $\mathrm{H} \delta, \mathrm{H} \gamma$, and $\mathrm{H} \beta$. Likewise, the template which provided the highest confidence $z_{X C}$ values for our sample is the composite quasar template from Vanden Berk et al. (2001). For 107 of our sources, $z_{X C}$ and $z_{E L}$ are consistent with each other within their errors. For the remaining 24 redshifts, 19 are from $z_{X C}, 4$ are from $z_{E L}$, and 1 was determined by hand. We note that for those sources with disagreeing values of $z_{X C}$ and $z_{E L}$, the poorer of the two values is clearly incorrect and not usable. Throughout the rest of this paper, we refer to the final redshifts produced by the 'spectrold' code as $z_{S D S S}$, and these values are listed in Table 3 .

\subsection{Redshifts from $\mathrm{Mg}$ II $\lambda 2800$}

In principle, individual emission lines can provide independent redshift estimates corresponding to the physical regions where those lines originated, e.g. the broad emission lines provide redshifts for the BLR. For our purposes, it is useful to know the redshift of the central SMBH (under the assumption of a single, active SMBH in the host galaxy) which can potentially be traced by the BLR if the gas is virialized. Fortuitously, given the redshift range of our sample, for all of our sources we have

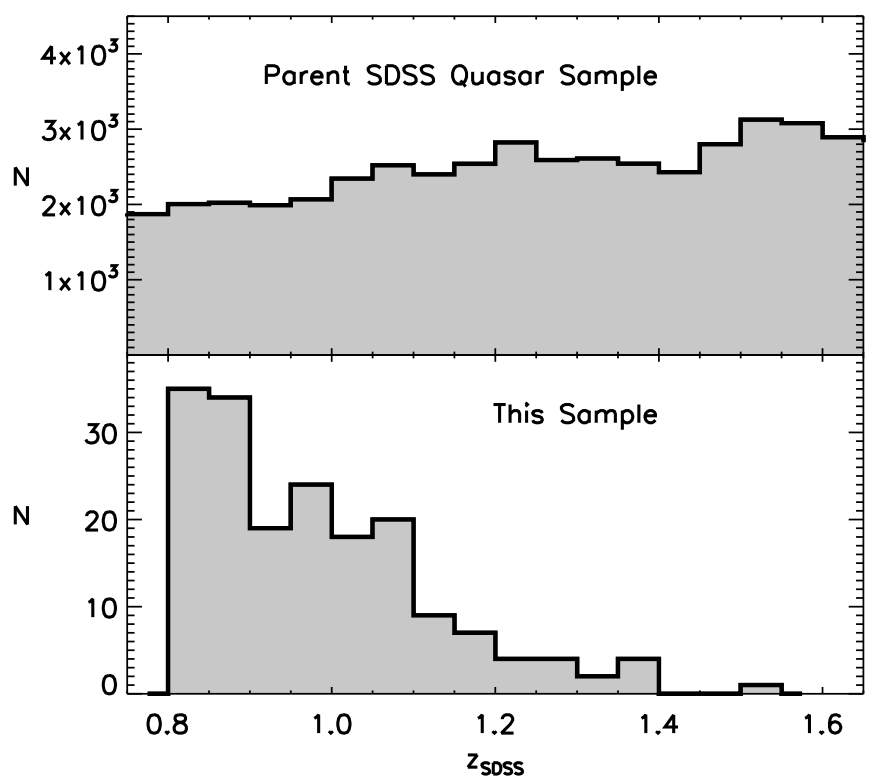

FIG. 5.- Top: Distribution of the parent SDSS quasar sample (Schneider et al. 2010) in the relevant redshift range. Bottom: Distribution of redshifts for our final double-peaked $[\mathrm{Ne} \mathrm{V}] /[\mathrm{Ne} \mathrm{III]}$ sample developed as described in Section [2 The cut-off below $z=0.80$ in our sample is entirely the result of our initial cut in redshift space (see Section 2.1), and the highest redshift for sources found through our selection process is $z=1.53$. The redshifts are described in Section 3

spectral access to the broad $\mathrm{Mg}$ II $\lambda 2800$ emission line, which has been shown to be virialized in the potential of the central SMBH (McLure \& Jarvis 2002). Therefore, we have used the Mg II $\lambda 2800$ emission line models from the catalog of SDSS DR7 quasar properties (Shen et al. 2011b) to obtain estimates of the BLR redshifts $\left(z_{M g I I}\right)$.

However, we would like to note several potential technical difficulties involved in measuring the $\mathrm{Mg}$ II $\lambda 2800$ line centroid which limit its application to our work. These difficulties include blending with Fe II emission, UV absorption features, and the often ambiguous presence of a narrow emission line component (which is actually the doublet $\mathrm{Mg}$ II $\lambda \lambda 2797,2802)$. We also note that the $\mathrm{Mg}$ II $\lambda 2800$ line centroids may be complicated if the low-ionization broad emission lines have the characteristic double-peaked profile of disk emitters (Eracleous \& Halpern 2003; Strateva et al. 2003). Furthermore, since we are considering the scenario of two AGN within the same host galaxy, we must consider the possibility that the broad Mg II $\lambda 2800$ line is a blending of two BLRs from two Type 1 AGN. Though the components of a dual AGN would not be close enough for the line-splitting to exceed the FWHM (several thousand $\mathrm{km} \mathrm{s}^{-1}$ ) and produce explicit double-peaked profiles (Shen \& Loeb 2010), two broad Mg II $\lambda 2800$ components separated by several hundred $\mathrm{km} \mathrm{s}^{-1}$ will still result in a relatively broadened, and possibly asymmetric, $\mathrm{Mg}$ II $\lambda 2800$ profile, thereby complicating the centroid measurement. We have visually inspected the $\mathrm{Mg}$ II $\lambda 2800$ line profile for each source in our sample in order to characterize their structure. There are several sources which show evidence for asymmetric structure, though it is generally difficult to discern if any of the profiles contain two broad components as would be 
the case if there are dual BLRs. Though many of those redshifts have rather large errors, a subset of them have robust line centroids with percent errors less than 1\% (73 sources) and percent errors less than $0.1 \%$ (52 sources). The Mg II $\lambda 2800$ redshifts are listed in Table 3.

\subsection{Comparison of $z_{S D S S}$ and $z_{M g I I}$}

Figure 4 shows the distribution of $\left(z_{S D S S}-z_{M g I I}\right) /(1+$ $\left.z_{S D S S}\right)$ where the $\mathrm{Mg}$ II $\lambda 2800$ redshifts appear to be systematically larger than the cross correlation redshifts in both our sample and the parent sample. This same effect is clearly apparent in the SDSS redshift analysis of Hewett \& Wild (2010), which suggests that we are seeing the same systematic trend in our analysis. While Hewett \& Wild (2010) provide redshifts which correct for this systematic offset in a statistical sense, these corrections may not be appropriate for some individual quasars. Therefore, we do not use these improved redshifts since our sample only contains 131 sources.

Based upon our comparison between $z_{S D S S}$ and $z_{M g I I}$, for the rest of our analysis we have chosen to use the $z_{S D S S}$ values and their associated errors. These SDSS redshifts will serve as the systemic velocities for our sources. All quoted line-splittings between the two line peaks $\left(\Delta V_{[\mathrm{NeV}]}\right.$ and $\left.\Delta V_{[\mathrm{NeIII}]}\right)$ and velocity offsets for the blue $\left(\Delta V_{[\mathrm{NeV}], \text { blue }}\right.$ and $\left.\Delta V_{[\mathrm{NeIII}], \text { blue }}\right)$ and red $\left(\Delta V_{[\mathrm{NeV}], \text { red }}\right.$ and $\left.\Delta V_{[\mathrm{NeII}] \text {,red }}\right)$ components are relative to these SDSS redshifts. The range of redshifts in our sample is $z=0.80$ (low-limit cutoff discussed in Section 2.1) to $z=1.53$ (highest redshift source in our sample). In Section 5.4 for which our analysis is heavily dependent upon the choice of redshift, we will also use the robust $z_{M g I I}$ measurements mentioned in Section 3.2 for comparison.

\section{GENERAL PROPERTIES OF THE SAMPLE}

In this section we describe several of the general properties of our sample. We compare the distributions of these properties (redshifts, $\mathrm{S} / \mathrm{N}$, and velocity offsets) to the parent sample or our low-redshift comparison sample in order to show our selection biases. Additionally, we discuss the results of our completeness estimates with respect to several of these and other properties.

\subsection{Distributions}

The SDSS redshifts we have adopted for our sample (Section 3) are shown and compared to the parent sample in Figure 5. The redshift distribution shows a peak near the low redshift cutoff of the sample, with a gradual decline out to higher redshifts, in contrast to the increasing population of the parent SDSS quasar sample. This strong dependence on redshift may reflect a dependence on equivalent width or a luminosity bias, which we discuss further below.

Figure 6] shows the distribution of the continuum S/N per pixel, where the mean values are 18.5 and 14.5 in the wavelength regions adjacent to $[\mathrm{Ne} \mathrm{V}] \lambda 3426$ and $[\mathrm{Ne}$ III $] \lambda 3869$, respectively. Compared to the parent sample, our sample shows a deficit at the low $\mathrm{S} / \mathrm{N}$ regime, and a stronger tail out to higher $\mathrm{S} / \mathrm{N}$ values. These distributions show that our selection is biased toward spectra of good quality which reflect the fact that detection of double-peaked structure in $[\mathrm{Ne} \mathrm{V}] \lambda 3426$ and

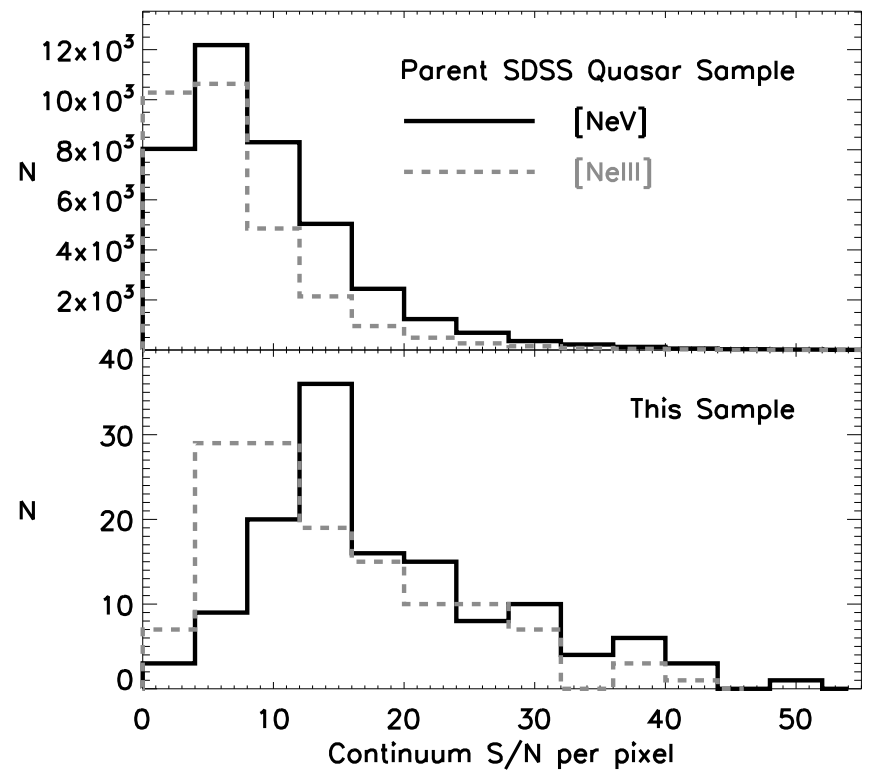

FIG. 6.- Distribution of continuum $\mathrm{S} / \mathrm{N}$ per pixel near the [Ne V] $\lambda 3426$ (black, solid line) and [Ne III] $\lambda 3869$ (gray, dashed line) emission lines for sources in our sample. For each distribution, the $\mathrm{S} / \mathrm{N}$ values were calculated over a set of featureless wavelength regions near the respective emission lines.

[Ne III $] \lambda 3869$ is sensitive to the $\mathrm{S} / \mathrm{N}$ and generally difficult at $\mathrm{S} / \mathrm{N}<5$. Furthermore, the larger mean value of the continuum $\mathrm{S} / \mathrm{N}$ near $[\mathrm{Ne} \mathrm{V}] \lambda 3426$ is reflective of the fact that $[\mathrm{Ne} \mathrm{V}] \lambda 3426$ is generally a weaker line than $[\mathrm{Ne}$ III] $\lambda 3869$ in AGN spectra Osterbrock \& Ferland 2006) and therefore a stronger signal is required for the detection of double-peaked structure. The dependence of our selection completeness on $\mathrm{S} / \mathrm{N}$ will be addressed below.

Since this study is motivated by the identification of high-redshift analogs of AGN with double-peaked [O III $] \lambda 5007$ lines, we compare the distribution of line properties of our sample to those of the [O III]selected comparison sample. The SDSS spectral resolution, $\mathrm{S} / \mathrm{N}$, and the intensity of the emission lines determine whether or not we are able to reliably detect double-peaks at a given separation in velocityspace. $[\mathrm{Ne} \mathrm{V}] \lambda 3426$ and $[\mathrm{Ne}$ III $] \lambda 3869$ are fainter than $[\mathrm{O}$ III $] \lambda 5007$ in AGN spectra by a factor of $\sim$ 4 (Ferland \& Osterbrock 1986), which means that selection based on $[\mathrm{Ne} \mathrm{V}] /\left[\begin{array}{ll}\mathrm{Ne} & \mathrm{III}\end{array}\right]$ will be biased toward larger line-splittings relative to $\left[\begin{array}{ll}\mathrm{O} & \mathrm{III}\end{array}\right] 5007$. For example, double-peaked samples selected through [O III] $\lambda 5007$ have sources with line splittings down to $\sim 200 \mathrm{~km} \mathrm{~s}^{-1}$, and the mean of the $[\mathrm{O}$ III $] \lambda 5007$ linesplittings in the Type 1 sample of Smith et al. (2010) is $\sim 420 \mathrm{~km} \mathrm{~s}^{-1}$. On the other hand, the mean of the $[\mathrm{Ne} \mathrm{V}] \lambda 3426$ and [Ne III] $\lambda 3869$ line-splittings of our sample is $\sim 700$ and $\sim 700 \mathrm{~km} \mathrm{~s}^{-1}$, respectively.

Figure 7 shows the distribution of velocity offsets from the systemic redshift for [Ne V] $] 3426$ and [Ne III] $\lambda 3869$ in both our sample and the [O III]-selected sample. While the measurements on the comparison sample were performed in the same way as for our sample (Section 2), these sources were selected from a sample of previously determined double-peaked AGN. Therefore, 


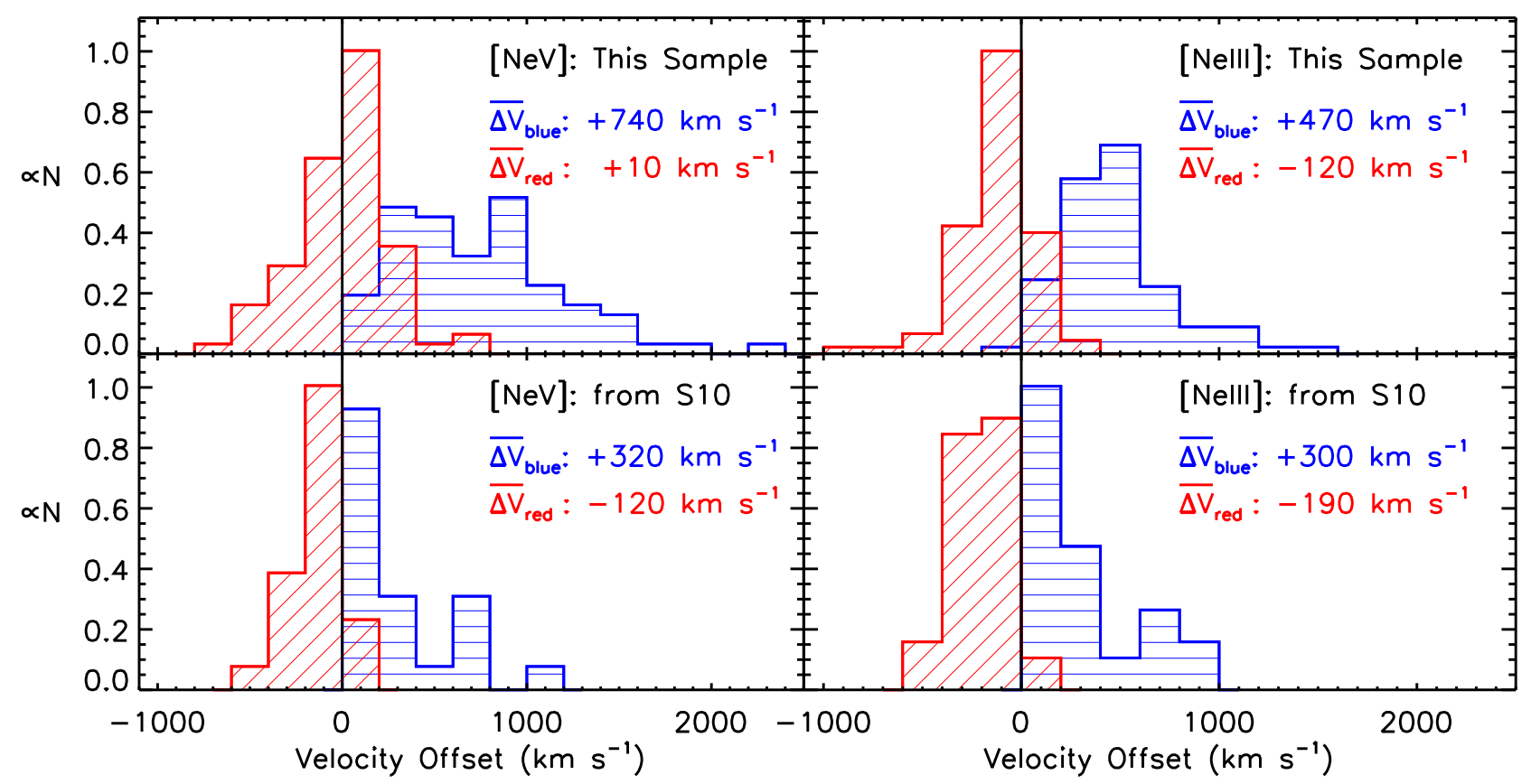

FIG. 7.- Distribution of velocity offsets for double-peaked AGN with positive velocities indicating blueshifts. 'Blue' component offsets $\left(\Delta V_{[\mathrm{NeV}], \text { blue }}\right.$ and $\left.\Delta V_{[\mathrm{NeIII}], \text { blue }}\right)$ are shown as horizontally-hatched blue lines, and 'red' component offsets $\left(\Delta V_{[\mathrm{NeV}], \mathrm{red}}\right.$ and $\left.\Delta V_{[\mathrm{NeIII}], \text { red }}\right)$ are shown as diagonally-hatched red lines. The peak value in each panel has been normalized to unity. Top: Sources selected through $[\mathrm{Ne} \mathrm{V}] \lambda 3426$ and [Ne III] 33869 emission line profiles. Bottom: Distribution of velocity-offsets for the [O III]-selected Type 1 AGN from the Smith et al. (2010) (S10) sample. All distributions are shown separately for [Ne V] $\lambda 3426$ (left) and [Ne III] $\lambda 3869$ (right). The average velocity-offsets are shown for each distribution. The black solid vertical line in each panel represents zero velocity offset.

we were able to more confidently identify two peaks in $[\mathrm{Ne} \mathrm{V}] \lambda 3426$ and $[\mathrm{Ne}$ III $] \lambda 3869$ at relatively smaller $\Delta V \mathrm{~s}$ for the [O III]-selected sample, as shown in Figure 7 . A Kolmogorov-Smirnov (KS) test indicates that the blue components of our sample come from a different distribution than those of the [O III $] \lambda 5007$-selected sample $\left(D_{[\mathrm{NeV}]}=0.56, D_{[\mathrm{NeIII}]}=0.43\right.$, and probabilities that they come from the same distribution $P_{[\mathrm{NeV}]}=3 \times 10^{-5}$, $\left.P_{[\mathrm{NeII}]}=7 \times 10^{-5}\right)$. A KS test also reveals a (less significant) difference in the red components $\left(D_{[\mathrm{NeV}]}=0.43\right.$, $D_{[\mathrm{NeIII}]}=0.33$, and probabilities of $P_{[\mathrm{NeV}]}=2.3 \times 10^{-3}$, $\left.P_{[\mathrm{NeIII}]}=5.6 \times 10^{-3}\right)$. This indicates that we are finding similar double-peaked narrow line sources to those selected through $[\mathrm{O}$ III $] \lambda 5007$ but that we are preferentially missing a portion of those with relatively small $\Delta V$ 's.

\subsection{Selection Completeness}

To estimate what fraction of sources with doublepeaked $[\mathrm{Ne} \mathrm{V}] \lambda 3426$ and/or [Ne III] $\lambda 3869$ we are missing due to the potential selection biases discussed above, we have estimated our selection completeness through simulations. Specifically, we generated artificial spectra designed to mimic SDSS spectra in the $[\mathrm{Ne} \mathrm{V}] /[\mathrm{Ne}$ III] wavelength ranges (a similar test was performed by Liu et al. 2010b for their [O III]-selected sample). Using the SDSS spectral dispersion, we modeled the continuum as a power-law and the emission lines as Gaussians with randomly assigned values for the parameters of equivalent width (EW), FWHM, continuum $\mathrm{S} / \mathrm{N}$ per pixel, and line offsets from the systemic velocity. The ranges of allowed values for those parameters were made uniform and at least slightly larger than the distributions of our final sample in order to ensure that we sampled the relevant parameter space for the analysis. While the EW distribution of our sample only extended to $\sim 35 \AA$ for the blue and red components, we allowed our simulations to have EWs of up to $50 \AA$ for the individual lines (100 $\AA$ total EW). The simulated FWHMs ranged from 100 to $1800 \mathrm{~km} \mathrm{~s}^{-1}, \sim 200 \mathrm{~km} \mathrm{~s}^{-1}$ larger than the largest FWHM of our sample. The simulated continuum $\mathrm{S} / \mathrm{N}$ values ranged from 2 to 33 , just larger than the range of our sample (see Figure 6). Finally, the simulated line offsets ranged from 0 to $2000 \mathrm{~km} \mathrm{~s}^{-1}$, $\sim 500 \mathrm{~km} \mathrm{~s}^{-1}$ larger than the largest line offsets of our sample. We generated a total of 10,000 spectra, with 2,000 of them having double-peaked emission lines, and the other 8,000 having single-peaked emission lines. Out of these simulated spectra, randomly distributed, we selected a final sample of double-peaked emission lines in the same manner as described in Section 2

We see a positive trend between completeness and EW, and find that we are highly incomplete over the EW range of our sample. For example, we estimate a completeness of just $\sim 1.2 \%$ for a total EW of $10 \AA$ and $\sim 4.5 \%$ for a total EW of $30 \AA$. We see a negative trend between completeness and FWHM which can be interpreted as the result of peak blending at large line widths. We see a moderate positive trend between completeness and $\mathrm{S} / \mathrm{N}$, with a completeness of $\sim 42 \%$ at $\mathrm{S} / \mathrm{N}=5$ and $\sim 50 \%$ at $\mathrm{S} / \mathrm{N}=30$. Finally, we see a strong dependence on velocity-splitting, with a completeness of $\sim 55 \%$ at $\Delta V=900 \mathrm{~km} \mathrm{~s}^{-1}$ and $\sim 34 \%$ at $\Delta V=500 \mathrm{~km} \mathrm{~s}^{-1}$. In contrast, Liu et al. (2010b) find a completeness of $\sim 75 \%$ 
at $\Delta V=900 \mathrm{~km} \mathrm{~s}^{-1}$ and $\sim 50 \%$ at $\Delta V=500 \mathrm{~km} \mathrm{~s}^{-1}$ for their [O III $] \lambda 5007$ sample.

Over comparable parameter ranges, we are less complete than Liu et al. (2010b) for all of the parameters ( $~ 50-80 \%$ of their completeness), with the exception of FWHM, for which we have an equivalent or slightly greater completeness at the large FWHM end of their distribution $\left(\sim 900 \mathrm{~km} \mathrm{~s}^{-1}\right)$. This is likely due to the fact that we allowed for a larger range of line offsets resulting in our ability to identify broader double peaks. The smaller completeness in our selection compared to those of Liu et al. (2010b) is likely due to the relatively smaller equivalent widths of $[\mathrm{Ne} \mathrm{V}] /[\mathrm{Ne} \mathrm{III}]$ allowed in our simulations which is reflective of the weaker intensities compared to $[\mathrm{O} \mathrm{III}] \lambda 5007$. Of the parameters investigated, we find that our completeness is most strongly dependent on and most drastically different from Liu et al. (2010b) in the velocity-offsets (Figure 7). This result is again consistent with the notion that we are preferentially missing a portion of those with relatively small $\Delta V \mathrm{~s}$.

Since false double-peaks are generated by noise, false positives are most likely to occur in low $\mathrm{S} / \mathrm{N}$ spectra in which random noise peaks are difficult to discern from the emission line signal. However, our conservative selection criteria effectively required that the $\mathrm{S} / \mathrm{N}$ be sufficiently large, as can be seen in Figure 6, with $S / N_{[\mathrm{NeV}]}<5$ for only 4 sources $(3 \%)$ and $S / N_{[\mathrm{NeIII}]}<5$ for only 11 sources $(8 \%)$. This is reflected in the fact that we did not select any false positives in our completeness analysis.

\section{CORRELATIONS AMONG SAMPLE PROPERTIES}

In the following section, we examine several trends related to the velocity offsets of the individual blue and red components from the systemic redshift and linesplittings between the blue and red components. We are interested in uncovering the prevailing mechanism(s) producing the double-peaked emission lines in our sample. In particular, we investigate the following four relationships: line-splitting vs line width, line-splitting vs quasar Eddington ratio, $[\mathrm{Ne} \mathrm{V}] \lambda 3426$ velocity offsets vs [Ne III] $\lambda 3869$ velocity offsets, and blue/red velocityoffset ratio vs blue/red luminosity ratio. In the following analyses we combine the $[\mathrm{Ne} \mathrm{V}] /[\mathrm{Ne}$ III] measurements of our sample with those of the [O III]-selected sample in order to increase both the sample size and the dynamic range of velocity offsets and line-splittings.

\subsection{A Correlation Between Line-Splittings and Line Widths}

In AGN with emission lines offset from the systemic velocity, there is often an observed positive correlation between the line peak offset (blueshifts being positive offsets) and line width. For example, in samples of so-called 'blue outliers' (AGN with [O III] $\lambda 5007$ lines blueshifted by $\left.>100 \mathrm{~km} \mathrm{~s}^{-1}\right)$, there is an observed positive correlation between the blueshift and line width of [O III] $]$ 5007 (Komossa et al. 2008). A similar trend was also seen by Liu et al. (2010b) for their sample of Type 2 double-peaked $[\mathrm{O}$ III $] \lambda 5007$ sources, with the offsets being the line-splitting between the two peaks. In a similar fashion, we have plotted the line splittings

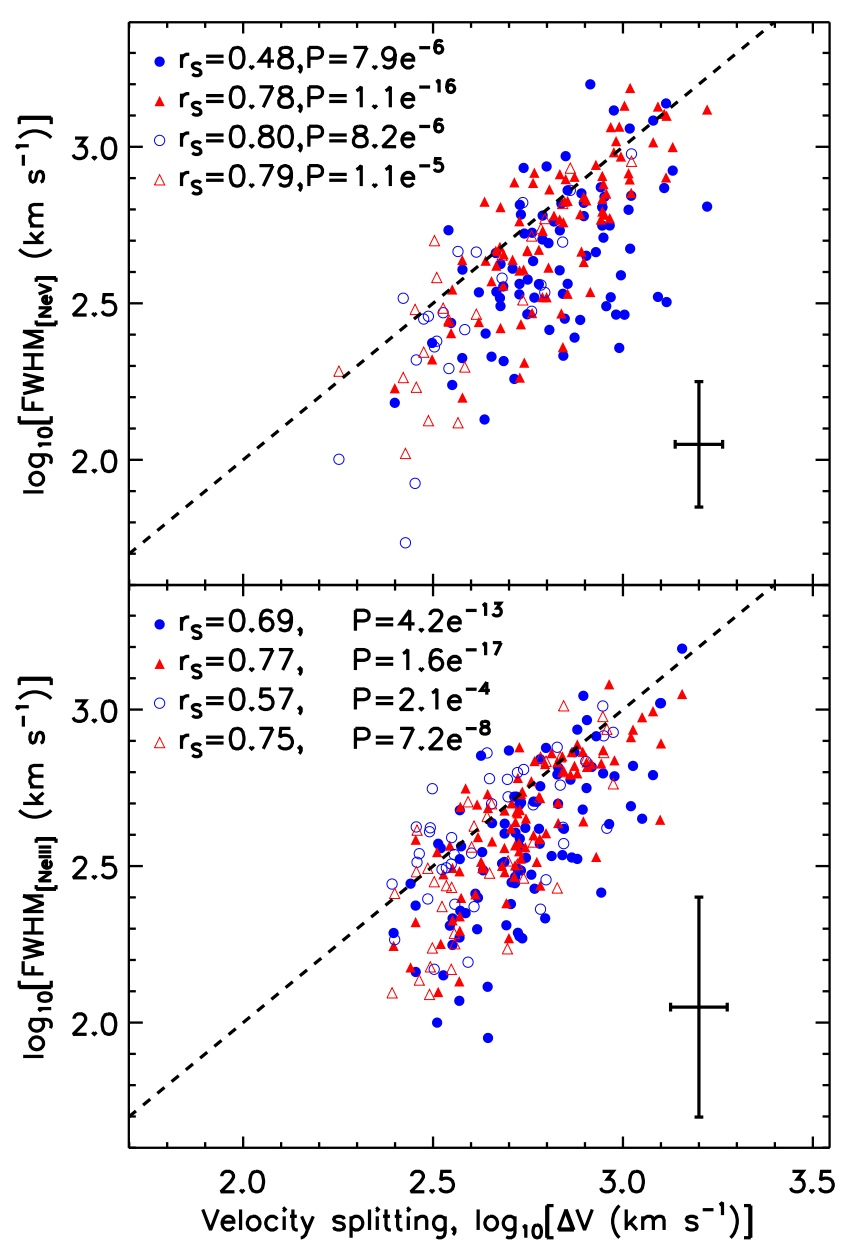

FIG. 8. - Plot of line width (FWHM) vs. line-splitting for $[\mathrm{Ne} \mathrm{V}] \lambda 3426$ (top panel) and [Ne III] $\lambda 3869$ (bottom panel). In each panel, the 'blue' and 'red' systems are labeled with blue circles and red triangles, respectively. Filled data points correspond to our $[\mathrm{Ne} \mathrm{V}] /[\mathrm{Ne} \mathrm{III}]$-selected sample, while unfilled points correspond to the [O III]-selected sample. The Spearman rank coefficients $\left(r_{S}\right)$ and probabilities of a null hypothesis $(P)$ are listed for the 'blue' and 'red' components. The dashed line is the one-to-one relation. The average $\mathrm{X}$ and $\mathrm{Y}$ errors are indicated in the lower right corner of each panel. Our completeness tests indicate that we are increasingly incomplete toward large-FWHM/small- $\Delta V$ (i.e. the upper left corners), but that we are increasingly complete toward small-FWHM/large- $\Delta V$ (i.e. the lower right corners).

against the line widths (FWHM) for the double-peaked $[\mathrm{Ne} \mathrm{V}] \lambda 3426$ and $[\mathrm{Ne}$ III] $\lambda 3869$ lines in both our sample and in the $[\mathrm{O}$ III]-selected sample (Figure 8). For both $[\mathrm{Ne} \mathrm{V}] \lambda 3426$ and [Ne III] $\lambda 3869$, statistically significant positive correlations between the line-splitting and FWHM are evident. The correlations are strong and the line-splittings are generally larger than the line widths, and both of these results were also found by Liu et al. $(2010 \mathrm{~b})$ with respect to [O III] $\lambda 5007$ emission lines.

The possible physical interpretations of these trends often includes an ionization stratification of the NLR in which the higher ionization lines originate closer to the ionizing radiation source (Zamanov et al. 2002; Komossa et al. 2008). In such a scenario, if there is a radially decelerating outflowing component of the NLR, then the higher ionization lines are accelerated to higher velocities. The emission lines produced in the inner por- 


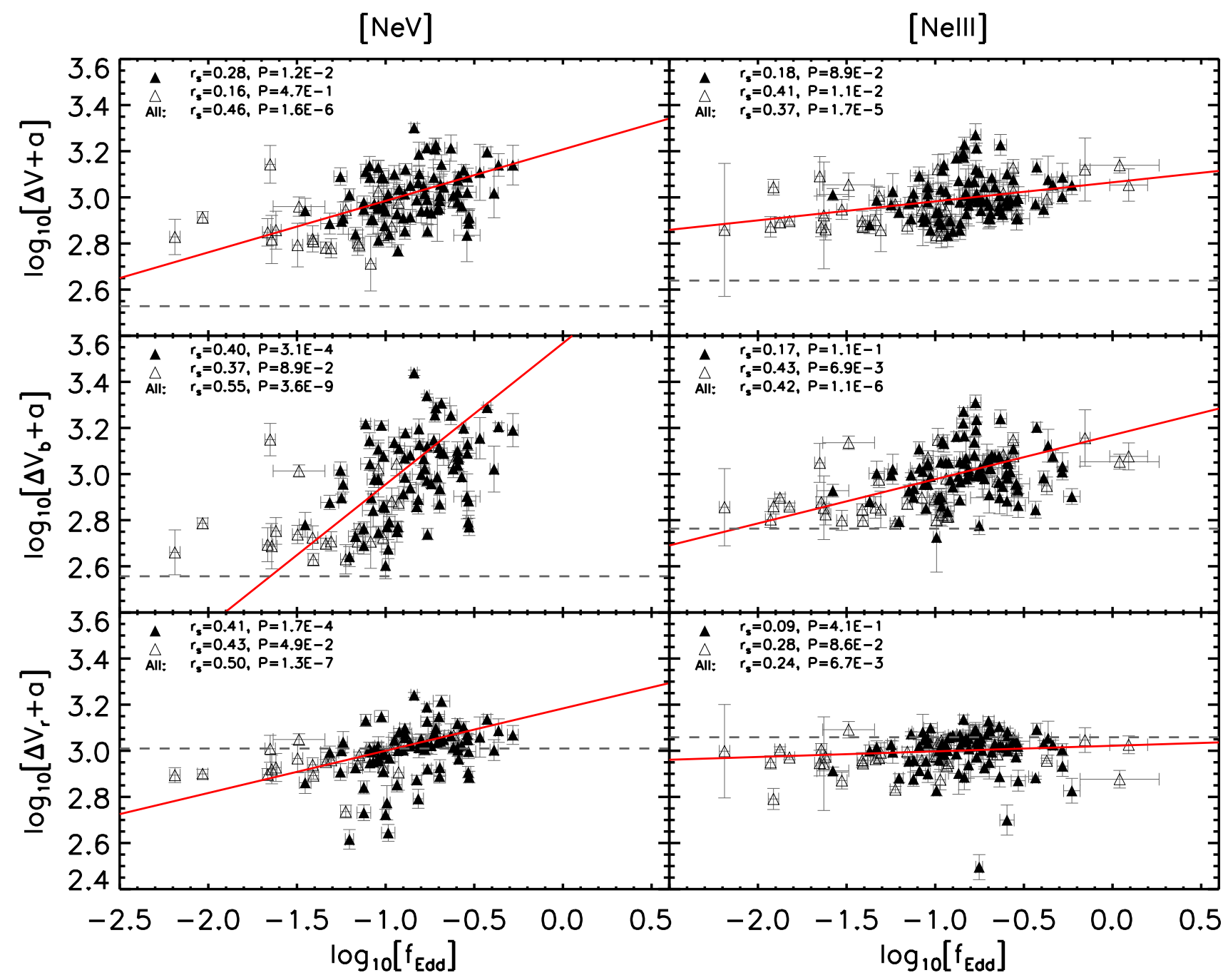

FIG. 9.- Plot of Eddington ratio versus velocity-splitting (top), blue component offsets (middle) and red component offsets (bottom) for $[\mathrm{Ne} \mathrm{V}] \lambda 3426$ (left) and [Ne III] $\lambda 3869$ (right). See Section 5.2 for details on calculations of $f_{\mathrm{Edd}}$. To be consistent among each subsample while accommodating the negative velocity-offsets of red components, the individual velocities for each distribution have been shifted by a constant, $a$, such that the mean of that particular distribution is equivalent to $1000 \mathrm{~km} \mathrm{~s}^{-1}$, i.e. $a=1000-(\overline{\Delta V})$. In each panel the horizontal gray, dashed line represents $\Delta V=0 \mathrm{~km} \mathrm{~s}^{-1}$ for that particular distribution. Sources at $0.8<z<1.6$ ([Ne V]/[Ne III]-selected) are plotted as filled triangles, and sources at $z<0.8$ ([O III $]$-selected) are plotted as unfilled triangles. The Spearman rank coefficients and probabilities of the null hypothesis are given for each of the sub-samples and for the combined samples. The red solid lines in each distribution are the best linear fits weighted by the $\mathrm{X}$ and $\mathrm{Y}$ errors (obtained with the 'FITEXY' routine) for the combined sample.

tions of the NLR will also be more broadened as their motion would be dominated by the bulge gravitational potential (Nelson \& Whittle 1996). Since the ionizing potentials of $[\mathrm{Ne} \mathrm{V}] \lambda 3426$ and $[\mathrm{Ne} \mathrm{III}] \lambda 3869$ (I.P. $=$ 41.07 and $97.16 \mathrm{eV}$, respectively) are larger than that of $[\mathrm{O}$ III] $] 5007(I . P .=35.15 \mathrm{eV})$, we might expect the correlation between line-splitting and FWHM to be stronger among these emission lines. This is also expected based on the higher critical electron densities for collisional de-excitation $\left(n_{\text {crit }}\right)$ of $[\mathrm{Ne} \mathrm{V}] \lambda 3426$ and $[\mathrm{Ne} \mathrm{III]}] \lambda 3869\left(n_{\text {crit }}=1.3 \times 10^{7} \mathrm{~cm}^{-3}\right.$ and $n_{\text {crit }}=9.5 \times 10^{6}$ $\mathrm{cm}^{-3}$, respectively) compared to $[\mathrm{O}$ III $] \lambda 5007\left(n_{\text {crit }}=\right.$ $6.8 \times 10^{5} \mathrm{~cm}^{-3}$ ) since electron densities will increase toward the nuclear region. Indeed, we find that the strengths of these correlations are generally comparable to or stronger than those for $[\mathrm{O}$ III] $]$ 55007 in the sample of Liu et al. (2010b) and in the narrow line Seyfert
1 (NLS1) sample from Komossa et al. (2008). These trends in our sample might be more reflective of the even stronger trends among the subset of 'blue-outliers' from Komossa et al. (2008).

\subsection{A Correlation Between Velocity-Splitting and Eddington Ratio}

Motivated by the outflow interpretation often used for samples of double-peaked emission line AGN, we investigate the relationship between the velocity-splittings and the quasar Eddington ratios $\left(f_{\text {Edd }}=L_{\text {bol }} / L_{\text {Edd }}\right)$. We calculated $L_{\text {Edd }}$ using the standard derivation of the Eddington luminosity, $L_{\mathrm{Edd}}=4 \pi c G M_{\mathrm{BH}} \mu_{e} / \sigma_{T}$, where $G$ is the gravitational constant, $\mu_{e}$ is the mass per unit electron, and $\sigma_{T}$ is the Thomson scattering cross-section (Krolik 1999). $M_{\mathrm{BH}}$ was estimated for each Type 1 AGN using the SMBH mass-scaling relationships from broad 

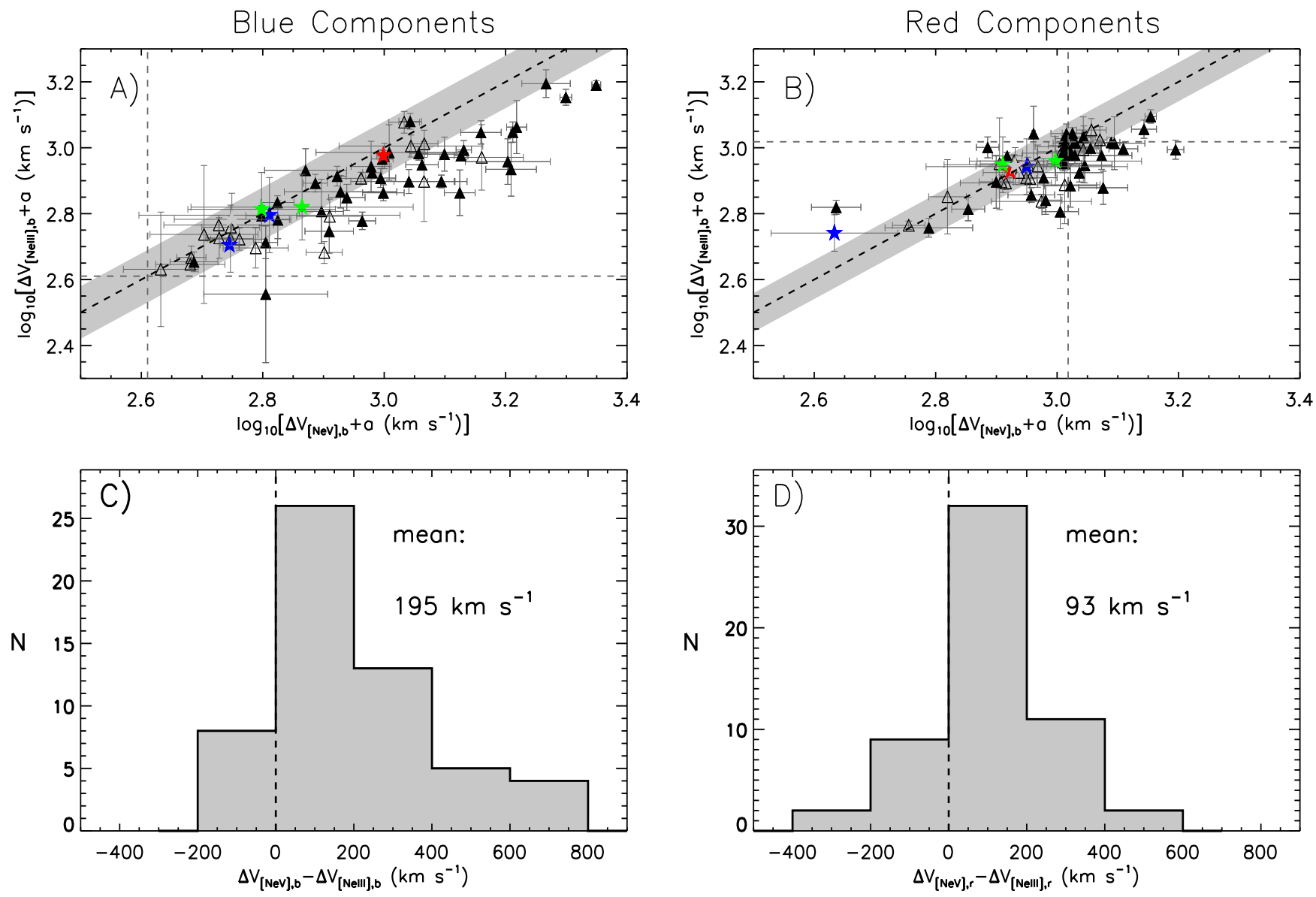

FIG. 10. - Top: Plots of $\Delta V_{\mathrm{blue},[\mathrm{NeV}]}$ versus $\Delta V_{\mathrm{blue},[\mathrm{NeIII}]}(\mathrm{A})$ and $\Delta V_{\text {red,[NeV] versus }} \Delta V_{\text {red, [NeIII] }}$ (B). The dashed line is the one-to-one relation. Each velocity has been shifted by the constant $a$ where $a=1000-\left(\overline{\Delta V_{[N e V]}}\right)$. In each panel the horizontal gray, dashed line represents $\Delta V_{[\mathrm{NeV}]}=0 \mathrm{~km} \mathrm{~s}^{-1}$ and the gray, shaded region represents the average of our sample. [Ne V]/[Ne III]-selected sources are plotted as filled circles while sources selected via [O III $] \lambda 5007$ are plotted as open circles. Additional sources are plotted as stars: extended NLRs (SDSS J110851.03+065901.4 and SDSS J135646.10+102609.0) and an unresolved NLR (SDSS J105052.46+083934.7) from Fu et al. (2012) are indicated by solid green and purple stars, respectively, [OIII]-selected dual AGN SDSS J171544.05+600835.7 (Comerford et al. 2011) and SDSS J150243.1+111557 (Fu et al. 2011b) are indicated by blue stars, and a [Ne V]/[Ne III]-selected dual AGN candidate from Barrows et al. (2012) is indicated by a red star (CXOXBJ142607.6+353351). Bottom: Histograms of $\Delta V_{[\mathrm{NeV}] \text {,blue }}-\Delta V_{[\mathrm{NeIII}], \text { blue }}(\mathrm{C})$ and $\Delta V_{[\mathrm{NeV}], \text { red }}-\Delta V_{[\mathrm{NeIII}], \text { red }}(\mathrm{D})$. The dashed line in the bottom panels represents $\Delta V_{[\mathrm{NeV}], \text { blue }}=\Delta V_{[\mathrm{NeIII}], \text { blue }}(\mathrm{C})$ and $\Delta V_{[\mathrm{NeV}], \text { red }}=$ $\Delta V_{[\mathrm{NeIII}], \text { red }}(\mathrm{D})$. The mean values are shown for each distribution.

emission line widths and monochromatic luminosities: $F W H M_{\mathrm{H} \beta}$ and $L 5100 \AA(z<0.8)$ or $F W H M_{\mathrm{MgII}}$ and $L 3000 \AA(z>0.8)$ from McClure and Dunlop (2004). The line widths are mostly from the SDSS DR7 catalog of quasar properties Shen et al. (2011b), though we visually inspected and re-fit several of them for which we were able to improve on the models. For each AGN with an estimate of $M_{\mathrm{BH}}$, we estimated $L_{\mathrm{bol}}$ from the monochromatic luminosities $L 5100 \AA(z<0.8)$ or $L 3000 \AA(z>0.8)$ and the bolometric corrections of Richards et al. (2006).

Figure 9 shows $f_{\text {Edd }}$ plotted against three quantities: velocity-splittings, blue velocity-offsets and red velocityoffsets. For both [Ne V] $\lambda 3426$ and [Ne III] $\lambda 3869$, a Spearman rank test reveals statistically significant correlations between $f_{\mathrm{Edd}}$ and each of these quantities. When comparing [Ne V] $\lambda 3426$ and [Ne III] $\lambda 3869$, it is apparent that the correlations are stronger for $[\mathrm{Ne} \mathrm{V}] \lambda 3426$ than for [Ne III] $\lambda 3869$ in all correlations tested. Additionally, for both [Ne V] $\lambda 3426$ and [Ne III] $\lambda 3869$ the correlations are strongest between $f_{\text {Edd }}$ and the blue velocity-offsets. We note that the low velocity-offsets are dominated by the [O III]-selected sources, which simply reflects the selection bias discussed in Section 4. If the velocity-splittings represent a NLR outflow in a significant number of these sources, then this result suggests that the radiation generated by the quasar accretion rate may play a crucial role in driving the line-splittings. Additionally, it appears that the blue component is more strongly dominated by outflowing material than the red component, which has implications for obscuration.

\subsection{Ionization Stratification}

The difference in I.P. between $[\mathrm{Ne} \mathrm{V}] \lambda 3426$ and [Ne III] $\lambda 3869$ is $\sim 56 \mathrm{eV}$ and can be used to provide insight on the gas dynamics in different regions of the NLR. Since the line offsets have been shown to correlate with the quasar Eddington ratios (Section [5.2), suggesting an outflowing component, we would like to search for evidence of an ionization stratification. Fig-

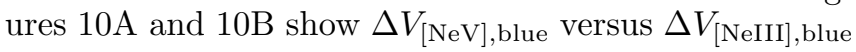




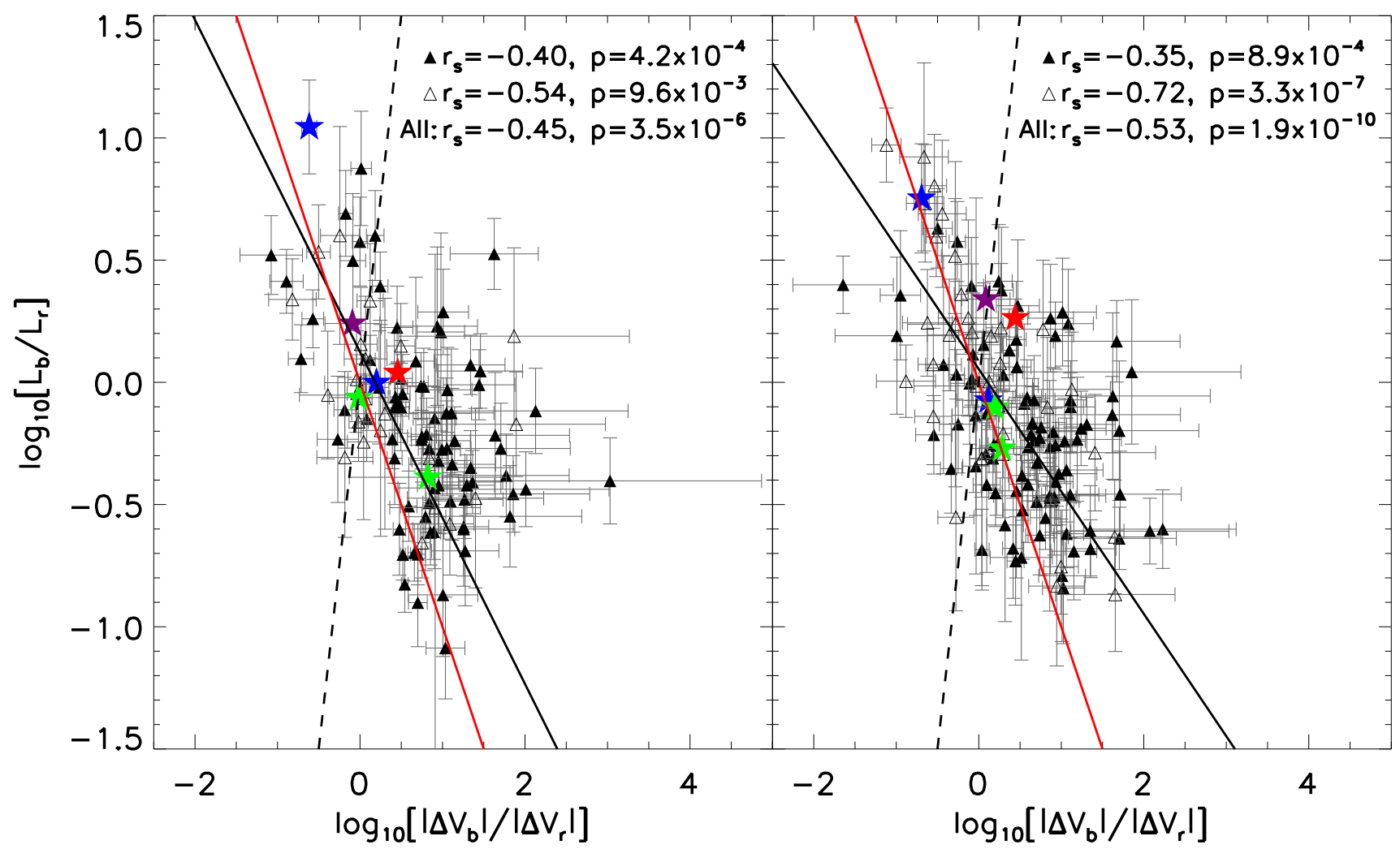

FIG. 11. - Plot of the velocity-offset ratio $\left(\left|\Delta V_{b}\right| /\left|\Delta V_{r}\right|\right)$ versus the luminosity ratio $\left(L_{b} / L_{r}\right)$ shown separately for [Ne V] $\lambda 3426$ (left) and $[\mathrm{Ne}$ III $] \lambda 3869$ (right). The solid black line is linear best fit weighted by the X and Y errors found using the 'FITEXY' routine. The solid red line is the theoretical relation assuming equivalent Eddington ratios for the blue and red components $\left(\epsilon_{1,2}=1\right)$, and the dashed black line is the theoretical relation for a bi-polar outflow, both of which are discussed in Section 5.4 The colored stars represent the same extended/unresolved NLRs, confirmed dual AGN and candidate dual AGN as in Figure 10 and have the same symbols. In each panel the Spearman rank correlations coefficients and probabilities of no correlation are shown for our sample, this comparison sample, and the combined ('All') sample.

and $\Delta V_{[\mathrm{NeV}], \text { red }}$ versus $\Delta V_{[\mathrm{NeIII}], \text { red }}$ for all sources in our sample and the [O III]-selected sample for which there are detectable double-peaks in both lines.

Though the true nature of the gas kinematics in NLRs is likely to be complex, under the simplest pictures of NLR ionization stratifications it is generally not expected that lines of lower ionization potential will show blue velocity-offsets larger than those of greater ionization potentials. Indeed, from Figure $10 \mathrm{~A}$ it can be seen that all of the points are consistent with having equivalent blue velocity-offsets in the two lines, or otherwise with a larger blue offset in $[\mathrm{Ne} \mathrm{V}] \lambda 3426$. This is also apparent in Figure $10 \mathrm{C}$, with a mean $\Delta V_{[\mathrm{NeV}] \text {,blue }}-\Delta V_{\text {[NeIII],blue }}$ value of $195 \mathrm{~km} \mathrm{~s}^{-1}$. Accounting for the $1 \sigma$ uncertainties, from our sample 15 sources $(40 \%)$ are consistent with equivalent blue velocity-offsets for both $[\mathrm{Ne} \mathrm{V}] \lambda 3426$ and [Ne III] $\lambda 3869$, and 23 sources $(60 \%)$ have significantly larger $[\mathrm{Ne} \mathrm{V}] \lambda 3426$ blue velocity offsets, consistent with the presence of an ionization stratification. Comparable fractions are seen in the $[\mathrm{O}$ III]-selected sample. The sources in the small velocity-offset portion of the plot are dominated by the [O III]-selected sample, which again reflects the selection bias discussed in Section 4. In contrast, the difference in the red offsets has a narrower distribution and a mean value more consistent with $\Delta V_{[\mathrm{NeV}], \mathrm{red}}=\Delta V_{[\mathrm{NeIII}], \mathrm{red}}($ Figures $10 \mathrm{~B}$ and [10 D). This suggests that the red systems are generally less stratified and have a different origin.

Also plotted are several additional double-peaked sources for which follow-up observations have been obtained, including extended and unresolved NLRs from Fu et al. (2012), dual AGN from Fu et al. (2012) and Comerford et al. (2011), and a candidate dual AGN from (Barrows et al. 2012). Note that the extended/unresolved NLRs and the dual AGN are [O III]selected, and the candidate dual AGN was selected based on $[\mathrm{Ne} \mathrm{V}] \lambda 3426$ and $[\mathrm{Ne} \mathrm{III}] \lambda 3869$. With the exception of the dual AGN SDSS J150243.1+111557, these additional sources are all consistent with no apparent ionization stratification in either the blue or red system by the $1 \sigma$ criteria we have used above.

\subsection{Dynamical Relation}

In this section we examine a dynamical argument, originally proposed by Wang et al. (2009), for the presence of dual AGN in our sample. Under the simplest picture of Keplerian orbital motion, a binary system of masses should show an inverse correlation between the ratio of their velocities, $V_{1} / V_{2}$, and the ratio of their masses, $M_{1} / M_{2}$, i.e. $V_{1} / V_{2}=M_{2} / M_{1}$. As shown in Wang et al. (2009) for their sample of double-peaked [O III] $] 5007$ AGN, the ratio of the line offsets is equivalent to the velocity ratio, and the mass ratio can be approximated by the luminosity ratio multiplied by a factor representing the ratio of the accretion rates, $\epsilon_{1,2}$. 
This yields the relation $L_{b} / L_{r}=\epsilon_{b, r} \Delta V_{r} / \Delta V_{b}$ between the blue and red components. For our sample, we have measured the requisite observational properties to perform this same analysis, but with the emission lines $[\mathrm{Ne} \mathrm{V}] \lambda 3426$ and $[\mathrm{Ne} \mathrm{III}] \lambda 3869$.

Figure 11 shows $\Delta V_{b} / \Delta V_{r}$ plotted against $L_{b} / L_{r}$ for both $[\mathrm{Ne} \mathrm{V}] \lambda 3426$ and $[\mathrm{Ne}$ III $] \lambda 3869$. The plots include both our sample and the low-redshift comparison sample. Also over-plotted are the same confirmed/strong dual AGN candidates and extended/unresolved NLR AGN shown in Figure 10 and discussed in Section 5.3. From a Spearman rank correlation test, it is clear that there is a strong negative correlation between $\Delta V_{b} / \Delta V_{r}$ and $L_{b} / L_{r}$, which is in the same sense as expected based on the theoretical relation of the binary, Keplerian orbit. Specifically, a strong correlation is seen individually in our sample, the comparison sample, and in the combined sample. For both [Ne V] $\lambda 3426$ and [Ne III] $\lambda 3869$, the comparison sample shows slightly stronger correlations than for our sample.

As argued in Wang et al. (2009), in a major merger one might expect that the SMBHs will be in similar environments and therefore, on average, should have similar Eddington ratios $\left(\epsilon_{b, r}=1\right)$. Therefore, we over-plotted the theoretical relation for $\epsilon_{b, r}=1$, which has the following form in base-10 logarithm space: $\log _{10}\left[L_{b} / L_{r}\right]=$ $\log _{10}\left[\epsilon_{b, r}\right]-\log _{10}\left[V_{b} / V_{r}\right]$. For both $[\mathrm{Ne} \mathrm{V}] \lambda 3426$ and $[\mathrm{Ne}$ III $] \lambda 3869$ the colored/starred sources are generally consistent with the theoretical and best-fit lines, and they span a large enough dynamic range to display the same general trend as our sample. The bestfit linear relations (weighted by the $\mathrm{X}$ and $\mathrm{Y}$ errors) are $\log _{10}\left[L_{b} / L_{r}\right]=(0.16 \pm 0.02)-(0.75 \pm 0.09) \times \log _{10}\left[V_{b} / V_{r}\right]$ $([\mathrm{Ne} \mathrm{V}] \lambda 3426)$ and $\log _{10}\left[L_{b} / L_{r}\right]=(0.05 \pm 0.02)-(0.51 \pm$ $0.04) \times \log _{10}\left[V_{b} / V_{r}\right]([$ Ne III $] \lambda 3869)$. These relations are similar to the theoretical relation for $\epsilon_{b, r}=1$, though they are technically not consistent when accounting for the $1 \sigma$ errors, with both fits yielding shallower slopes. The shallower slopes appear to be caused by some of the sources at the low $L_{b} / L_{r}$ end which extend out to larger $V_{b} / V_{r}$ ratios than the rest of the sample. In this portion of the plot, our sources are systematically shifted above the theoretical relation. Finally, we have over-plotted the theoretical relation expected for a biconical outflow, $L_{b} / L_{r}=\left(\Delta V_{b} / \Delta V_{r}\right)^{3}$ (developed in Wang et al. 2009). This relation is in the nearly the opposite sense as the for the binary relation and is far from agreeing with the best-fit relations. In Section 7.2.3 we will discuss how this correlation and the offset from the theoretical relation may be consistent with the presence of both dual AGN and outflows in our sample.

While the version of this analysis presented in Wang et al. (2009) utilized host galaxy redshifts (obtained through fitting template galaxy spectra), we can not obtain that information for our sample since the quasar continuum outshines the galaxy starlight. Instead, the velocity offsets used in our analysis here are relative to the SDSS redshifts $\left(z_{S D S S}\right)$ discussed in Section 3. However, we have attempted to investigate the dependence of our results on the choice of redshift. Recalling our discussion in Section 3. we also have redshifts based upon $\mathrm{Mg}$ II $\lambda 2800$ which (in the single SMBH scenario) should trace the central, active SMBH redshift. Therefore, we examined $V_{b} / V_{r}$ versus $L_{b} / L_{r}$ for the subset of our sample with robust (percent error $<0.1 \%) z_{M g I I}$ values. We find that this does not introduce a significant change in the best-fit coefficients for $[\mathrm{Ne} \mathrm{V}] \lambda 3426$ or $[\mathrm{Ne} \mathrm{III}] \lambda 3869$.

\section{RADIO LOUDNESS}

Since $91 \%(119 / 131)$ of our sources are in the FIRST footprint $(5 \sigma$ flux limit of $\sim 750 \mathrm{mJy}$ ), to determine their radio-loudness we have adopted the commonly used definition of radio to optical luminosity ratio $\mathcal{R}=$ $L 5 \mathrm{GHz} / L 2500 \AA$, with $\mathcal{R}=10$ being the cutoff value for the radio-loud classification ( $\mathrm{Ho}$ 2002). From our parent sample, the fraction of radio-loud SDSS quasars in the FIRST footprint is $10 \%$, whereas that fraction is $23 \%$ in our final double-peaked [Ne V]/[Ne III] sample. For comparison, Smith et al. (2010) obtained 9\% (parent sample at $z<0.8$ ) and $27 \%$ (double-peaked [O III] $\lambda 5007$ sample), respectively. This preferential selection of radio sources over the parent quasar population in both studies suggests that the origin of the double-peaks might be related to the presence of radio jets in some sources, as in the case of SDSS J151709.20+335324.7 (Rosario et al. 2010)

\section{INTERPRETATION}

In this section, we synthesize the results of the previous sections to highlight the most likely physical scenarios that produce the line splitting and line offsets in our sample of quasars with high-ionization, double-peaked narrow emission lines. In particular, we examine the possibilities of AGN outflows and dual AGN.

\subsection{Examining the Outflow Hypothesis}

The correlation between line-splitting and line width evident in the blue and red systems of both $[\mathrm{Ne} \mathrm{V}] \lambda 3426$ and [Ne III] $\lambda 3869$ (Figure 8) indicates that the mechanisms producing both the line-splittings and the line broadening are related, an observation which is consistent with the two emission line components originating near the same AGN. In general, emission line velocity-offsets from the host galaxy or quasar redshift are often interpreted as evidence for outflowing photoionized gas; powerful AGN, including quasars, are known to be capable of driving high-velocity and/or large-scale outflows (Fischer et al. 2011). We will discuss how properties of our sample are consistent with some of the mechanisms known to produce outflows, and how they result in stratified NLRs. Furthermore, we will discuss our interpretation within the context of a proposed outflow and stratification geometry shown in Figure 12.

\subsubsection{Mechanisms Producing AGN Outflows}

Two of the commonly proposed mechanisms for driving outflows in powerful AGN are radiation pressure from the accretion disk and radio jets. Figure 9 shows that the velocity-splittings are correlated with the quasar Eddington ratio, $f_{\mathrm{Edd}}$, for $[\mathrm{Ne} \mathrm{V}] \lambda 3426$ and for $[\mathrm{Ne} \mathrm{III}] \lambda 3869$. This result is consistent with the notion that outflows can be driven by radiation pressure from an accretion disk, and that more actively accreting SMBHs will drive stronger outflows. This scenario is shown in Figure 12 with the radiation emanating from the accretion disk in the commonly assumed bi-conical shape Antonucci 


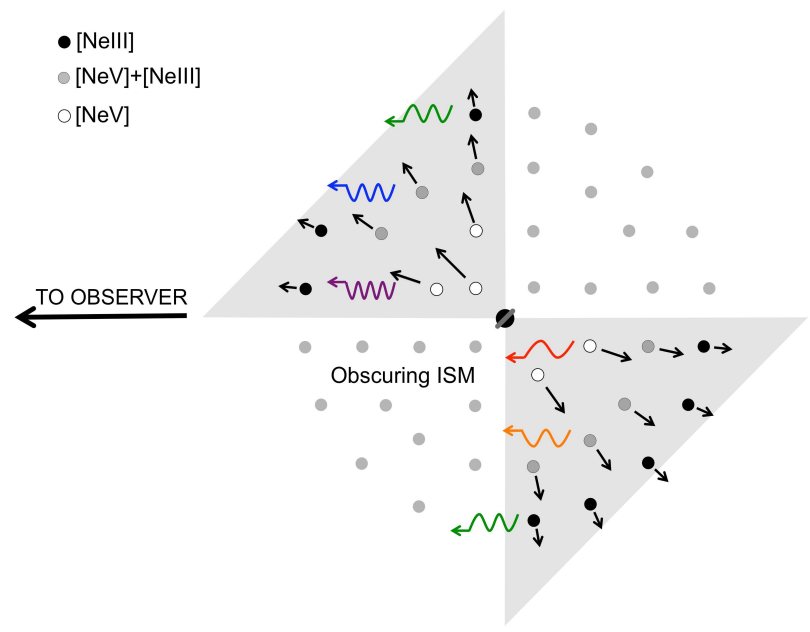

FIG. 12.- Cross-section of the NLR showing our proposed geometry of the outflow/ionization stratification scenario and the relative origins of $[\mathrm{Ne} \mathrm{V}] \lambda 3426$ and [Ne III] 33869 . The ISM around the central AGN is assumed to be spherically symmetric, the orientation of the outflow axis is $45^{\circ}$ from face-on, and we only show the NLR out to a radius which includes all of the [Ne V] $\lambda 3426$ and [Ne III] $\lambda 3869$ emission. The bi-cone of ionizing radiation (with an opening angle of $45^{\circ}$ ) emanating from the central SMBH is indicated by the gray shaded regions. Various individual portions of the ISM and NLR are indicated by solid circles. Portions where $[\mathrm{Ne} \mathrm{V}] \lambda 3426$ is produced but not [Ne III] $\lambda 3869$ are shown as solid white circles, portions where both [Ne V] $\lambda 3426$ and [Ne III] $\lambda 3869$ are produced are indicated by solid gray circles, and portions where $[\mathrm{Ne} \mathrm{III}] \lambda 3869$ is produced but not $[\mathrm{Ne} \mathrm{V}] \lambda 3426$ are indicated by solid black circles. Portions of the ISM not in the ionization cone are indicated by light gray circles. The black arrows indicate the velocities relative to the central SMBH, and the colored waves/arrows represent Doppler shifting of the light due to the velocity component along the line of sight to the observer.

1993). Our result is also consistent with the results of Komossa et al. (2008) who find that their sample of NLS1s with offset [O III $\lambda 5007$ lines have relatively large Eddington ratios which might be reflective of the radiation driving the outflow. Finally, that the correlation with $f_{\text {Edd }}$ is stronger for $[\mathrm{Ne} \mathrm{V}] \lambda 3426$ than for [Ne III] $\lambda 3869$ fits with a picture in which the NLR gas closest to the central engine is more strongly accelerated by the radiation pressure, as indicated in Figure 12 by the larger velocity vectors on material closest to the SMBH.

However, it is possible that not all of the outflows are driven by radiation pressure. In particular, radio jets are known to drive outflows in AGN by entrainment of NLR material, and our sample (and the [O III]-selected sample) have radio loud fractions of $\sim 25 \%$ (Section 6). Therefore, the high fraction of radio loud quasars in our sample relative to the parent quasar sample is a strong indication that the double-peaked sample we have compiled includes quasars with outflowing components.

\subsubsection{Evidence for Stratified NLRs}

If outflows are a common mechanism for driving linesplitting in NLRs, then this should naturally result in a stratified NLR since lines of greater I.P. and $n_{\text {crit }}$ will be preferentially produced nearer to the AGN where they are accelerated to higher velocities relative to lines of lower I.P and $n_{\text {crit }}$. The relative origins and velocities of emission lines in the stratification scenario are illustrated in Figure 12, where [Ne V] $\lambda 3426$ originates closer to the SMBH than $[\mathrm{Ne}$ III] $\lambda 3869$. We examined this scenario by looking at the relationship between the velocityoffsets of $[\mathrm{Ne} \mathrm{V}] \lambda 3426$ and [Ne III] $\lambda 3869$ in Section 5.3. In that analysis, Figure [10A shows that $60 \%$ of the sources have a significantly larger blue velocity offset in $[\mathrm{Ne} \mathrm{V}] \lambda 3426$ compared to [Ne III] $\lambda 3869$, suggestive of a stratified NLR. We are only seeing the projected velocityoffsets, so this percentage might represent a lower limit on the number of sources with stratified NLRs when accounting for random orientations of the outflow axes. We note that in Figure 10 the largest stratifications are seen at the largest velocities. This could also be a result of projection effects, since orientations which reduce the radial velocity components will also reduce the observed stratification. However, this trend may also be due, in part, to the physical effect of stronger outflows (larger velocities) producing larger stratifications.

For most of the sources in our sample we can only place upper limits on the fluxes and velocity offsets of blue [O II $] \lambda 3727$ components. This is partly due to the blending of the $\lambda 3726,3729 \AA$ doublet (Section 2.2). However, if the double emission line components are driven by outflows and the NLR is stratified, one might expect that [O II $] \lambda 3727$, with a relatively small ionization potential of I.P. $=13.614 \mathrm{eV}$ and critical density of $n_{\text {crit }}=3.4 \times 10^{3}$ $\mathrm{cm}^{-3}$, will be strongest in a portion of the NLR relatively further from the central AGN. As a result, it will not be accelerated to high velocities, resulting in a small line-splitting.

With the outflow axis oriented at some angle intermediate to edge-on or face-on, the redshifted NLR emission will consequently be more attenuated than the blueshifted emission, as illustrated by the 'obscuring ISM' labeled in Figure 12. The portions of the NLR with the largest line of sight velocity components will be most obscured. Conversely, the portions with the smallest line of sight velocity components will be the least obscured. The result is that, in the presence of such attenuation, the most redshifted portion of the emission lines will obscured, moving the observed position of the red emission peak closer to the systemic, i.e. non-Doppler shifted, redshift. In this case the red component resembles the 'classical' NLR. This is consistent with observations in which offset narrow emission lines in AGN are usually blueward of the systemic velocity, indicating that we are able to view the outflowing component moving toward the observer, while the component moving away from the observer is obscured by a larger column of dust. For example, from Figure 7 it appears that the mean magnitude of the red component velocity-offsets from the systemic redshift are generally smaller than those of the blue components, consistent with the notion that the red components are less dominated by outflows.

Note that in Figure 12, with sufficient attenuation even the NLR emission which is least Doppler-shifted will be obscured, resulting an apparent blueshifting of the red component, as is occasionally seen in some of our sources and in other studies (Spoon \& Holt 2009). For example, the blue $[\mathrm{Ne} \mathrm{V}] \lambda 3426$ component may be emitted from a portion of the NLR on the observer's side which is closest to the central source and moving at the greatest velocity (e.g. white dots in Figure [12), while the red $[\mathrm{Ne} \mathrm{V}] \lambda 3426$ component is from a portion which is 
further from the central source (e.g. grey dots in Figure 12). We did find in Section 5.2 evidence for a mild positive correlation between the red line offsets and the quasar Eddington ratio. This suggests that, while the red system tends to represent the 'classical' NLR, it is still effected by the radiation pressure since it must originate close enough to the central source where the ionizing flux is sufficient. Additionally, Figures $10 \mathrm{~B}$ and $10 \mathrm{C}$ show that there is some evidence for stratification of the red systems (though much less significant than for the blue systems).

\subsection{Implications for Dual AGN at High-Redshift}

It is possible that some of the sources in our sample may host two SMBHs following a galaxy merger. In this case, the double-peaks may be from two distinct NLRs that each accompanies its own active SMBH, or perhaps two NLR peaks are influenced by the orbital motion of two SMBHs (Blecha et al. 2012). So far there are only a handful of known plausible merger remnants hosting two AGN at redshifts comparable to our sample: $z \sim 0.709$ (Gerke et al. 2007), $z \sim 0.78$ (Comerford et al. 2009b), and $z \sim 1.175$ (Barrows et al. 2012). Since galaxy mergers were more frequent at higher redshifts, we would like to investigate the dual AGN scenario for the sources in our sample.

\subsubsection{Sources with No Apparent Ionization Stratification}

The two [O III]-selected confirmed dual AGN for which we measured double peaks in $[\mathrm{Ne} \mathrm{V}] \lambda 3426$ and $[\mathrm{Ne}$ III] $\lambda 3869$ are consistent with no apparent ionization stratification (Figure10). Additionally, there is only one candidate dual AGN identified through the doublepeaked profile of $[\mathrm{Ne} \mathrm{V}] \lambda 3426$ and [Ne III] $\lambda 3869$, and it is also consistent with no apparent ionization stratification (Barrows et al. 2012). In these sources, the evidence for outflowing NLR material is less compelling, and the line-splitting may instead be produced by orbital motion of two AGN about each other. Likewise, the $40 \%$ percent of our sources plotted in Figure 10 with no apparent ionization stratification may include cases where the double-peaks are the result of two SMBHs following a galaxy merger. Additionally, we see explicit double [O II] $\lambda 3727$ peaks for a subset (11) of our sources which perhaps suggests that the outflow scenario is less likely in these sources since $[\mathrm{O}$ II $] \lambda 3727$ should originate at a greater distance from the central AGN, as mentioned in Section 7.1 .

We note, however, that the lack of an apparent ionization stratification does not preclude the possibility of an outflow, or general gas kinematic origin of the double-peaked emission lines. For example, the two extended NLR AGN from Fu et al. (2012) have no measurable ionization stratification but the double emission components are known to be produced by the NLR around a single AGN based on integral-field spectroscopy and high-resolution imaging. Conversely, a stratified NLR (or two stratified NLRs) does not preclude the presence of two AGN. For example, as discussed in Section 5.3, our measurement of $[\mathrm{Ne} \mathrm{V}] /[\mathrm{Ne} \mathrm{III}] \lambda 3869$ in the confirmed dual AGN SDSS J150243.1+111557 shows some evidence for a stratification.

\subsubsection{Dual AGN with Large Velocities}

As is evident from Figure 7 and discussed in Section 4 the velocity-splittings in our sample are generally larger than those from [O III]-selected samples, which might tend to select against likely dual AGN candidates at kpcscale separations since they would not be bound to the merging galaxy system with such large velocities. Most strong dual AGN candidates have $\Delta V$ s less than 500 $\mathrm{km} \mathrm{s}^{-1}: \Delta V=150 \mathrm{~km} \mathrm{~s}^{-1}$ (Comerford et al. 2009a; Civano et al. 2010), $\Delta V=500 \mathrm{~km} \mathrm{~s}^{-1}$ (Xu \& Komossa 2009), $\Delta V=350 \mathrm{~km} \mathrm{~s}^{-1}$ (Comerford et al. 2011), and $\Delta V=420 \mathrm{~km} \mathrm{~s}^{-1}$ (McGurk et al. 2011; Fu et al. 2012).

However, there are several candidates with velocities $>500 \mathrm{~km} \mathrm{~s}^{-1}: \Delta V=630 \mathrm{~km} \mathrm{~s}^{-1}$ (Gerke et al. 2007) and $\Delta V=700 \mathrm{~km} \mathrm{~s}^{-1}$ (Barrows et al. 2012). Additionally, the dual AGN hypothesis could be allowed for larger $\Delta V$ s if the AGN pairs are at small separations. For example, Blecha et al. (2012) find in their simulations that large $\Delta V \mathrm{~s}\left(>500 \mathrm{~km} \mathrm{~s}^{-1}\right)$ are often associated with dual AGN at sub-kpc separations during pericentric passages. For comparison, $23 \%$ of our sample have $\Delta V<500 \mathrm{~km}$ $\mathrm{s}^{-1}, 45 \%$ have $500<\Delta V<800 \mathrm{~km} \mathrm{~s}^{-1}$, and $32 \%$ have $\Delta V>800 \mathrm{~km} \mathrm{~s}^{-1}$, with a maximum of $\Delta V=1665 \mathrm{~km}$ $\mathrm{s}^{-1}$. Therefore, though a fraction of our sample exhibit $\Delta V \mathrm{~s}$ higher than expected for dual AGN, $2 / 3$ fall in the range expected for either kpc or sub-kpc separation AGN pairs. We note that recent numerical simulations suggest dual activation of the SMBHs following a galaxy merger is most likely to occur at separations smaller than 1-10 kpc (Van Wassenhove et al. 2012). Therefore, under this picture of dual activation, the correlation between Eddington ratio and line-splitting seen in Figure 9 would naturally emerge for a sample of dual AGN.

As discussed in Section 3, for dual AGN with sufficiently large orbital velocities the broad emission line profiles may be significantly broader than expected if both components are Type 1 AGN. To test for such additional broadening, we have compared the Mg II $\lambda 2800$ FWHMs with those of Fe II, but find no evidence for systematically broadened Mg II $\lambda 2800$ compared to Fe II. Furthermore, a K-S test does not indicate a significant difference between the Mg II $\lambda 2800 \mathrm{FWHM}$ distribution of our sample and that of the parent sample. However, we note that the mean $F W H M_{\text {MgII }}$ value for our sample $\left(4740 \mathrm{~km} \mathrm{~s}^{-1}\right)$ is slightly larger than that of the parent sample $\left(4580 \mathrm{~km} \mathrm{~s}^{-1}\right)$ which is perhaps suggestive of additional Mg II $\lambda 2800$ broadening.

\subsubsection{Testing the Dynamical Argument}

In Section 5.4 we tested a dynamical argument for the presence of dual AGN in our sample. The results are generally consistent with the theoretical expectation for a binary, Keplerian orbit (Figure 11). However, to understand the extent to which we can interpret this result, we must also strongly consider the role of alternative physical scenarios in producing such a correlation.

First, we note that for both $[\mathrm{Ne} \mathrm{V}] \lambda 3426$ and [Ne III $] \lambda 3869$, based on the Spearman rank test, the comparison sample shows slightly stronger correlations than for our sample. If this is an indication that the comparison sample shows stronger evidence for dual AGN, it would be consistent with the notion that larger velocitysplittings are less likely to be associated with dual AGN 
since our sample has larger velocity-splittings than the comparison sample.

Second, it is worth noting that the coefficients for the best-fit linear relations of $[\mathrm{Ne} \mathrm{V}] \lambda 3426$ and $[\mathrm{Ne}$ III $] \lambda 3869$ are in disagreement. This is consistent with the correlation being produced by outflows (or at least some of the sources experiencing outflows) since the red component of [Ne III] $\lambda 3869$ would originate at a greater distance from the observer (compared to the red $[\mathrm{Ne} \mathrm{V}] \lambda 3426$ component) and therefore be even more obscured relative to the blue component (Figure 12). In this case, the $L_{b} / L_{r}$ ratio should be even larger for $[\mathrm{Ne}$ III $] \lambda 3869$, making the slope shallower as observed.

However, it is possible that if our sample contains some combination of outflows and dual AGN then the outflows are responsible for deviations from the theoretical binary relation. For example, as discussed in Section 5.4 many of the sources at the low $L_{b} / L_{r}$, high $V_{b} / V_{r}$ portion of Figure 11 are offset above the theoretical relation. These sources may be more likely to represent outflows since they are trending in the same direction as the outflow relation. Additionally, they have large $V_{b} / V_{r}$ ratios because the red component is near the systemic redshift, consistent with attenuation of the redshifted outflow component. Lastly, the $L_{b} / L_{r}$ ratios are smaller, indicating that the red component is stronger, and the blue component is a lower luminosity, extended wing as is often seen in outflows and is seen a few of our sources. If these deviant sources are most likely to be outflows, then the remainder would be more consistent with the theoretical relation. Additionally, the remainder would have a $L_{b} / L_{r}$ distribution consistent with $\epsilon_{b, r}=1$, similar to the result of Wang et al. (2009).

We note that an additional source of scatter in the correlation could be due to stochastic accretion, such that the luminosity ratio does not accurately reflect the true mass ratio. This effect could be particularly significant when the SMBHs are at larger separations when gas is less efficiently funneled to the nuclear regions. Interestingly, at $z=0.8-1.6$, the 3" fiber diameter of the SDSS spectrograph corresponds to $\sim 22-25 \mathrm{kpc}$, so that our sample may contain such early-stage mergers.

\subsubsection{Estimating the Fraction of Dual AGN in Our Sample}

While the completeness of our selection process as discussed in Section 4 suggests that there is a significant number of double-peaked emitters that we have missed, especially at small $\Delta V \mathrm{~s}$, we can not correct for the true number since we do not know the shape of the underlying distribution. However, at the least we can use our [O III]selected comparison sample to estimate what fraction of $[\mathrm{O}$ III $] \lambda 5007$ double-peaked emitters that we missed based on selection through $[\mathrm{Ne}$ V $] \lambda 3426$ or $[\mathrm{Ne}$ III] $\lambda 3869$. Of the 57 Type 1 AGN from (Smith et al. 2010), we could reliably measure double [Ne III] $\lambda 3869$ peaks for $67 \%$ (we note that this fraction is consistent with the comparison between our completeness estimates and those of Liu et al. (2010b) in Section 4.2). Based on this fraction, we arrive at a corrected number of 195 double-peaked [O III] $\lambda 5007$ sources and a double-peaked [O III $\lambda 5007$ AGN fraction of $\sim 0.5 \%$ at $0.8<z<1.6$. This fraction is likely to be a lower estimate because the double-peaked [Ne V] $\lambda 3426$ and [Ne III] $\lambda 3869$ lines of the [O III]-selected sample were not selected in exactly the same way (i.e. we had a prior knowledge of the double-peaked separation). Therefore, the doublepeaked $[\mathrm{O}$ III] $\lambda 5007$ AGN fraction at $0.8<z<1.6$ is likely to be larger, potentially making it consistent with the fraction of $\sim 1 \%$ found by Liu et al. (2010b) at $z<0.8$.

To actually estimate the expected fraction of dual AGN in our sample we need the true fractions of double-peaked AGN and of dual AGN out of all AGN at $0.8<z<1.6$, neither of which are known. However, we may make several reasonable assumptions that provide a rough estimate. First, in order to determine the true fraction of double-peaked AGN out of all AGN at $0.8<z<1.6$, we need to correct for both our selection incompleteness and random projection effects. This fraction was estimated by Shen et al. (2011a) in which they determined that the fraction of detectable double-peaked [O III] $\lambda 5007$ AGN is only $20 \%$ of the actual number of AGN with double $[\mathrm{O}$ III $] \lambda 5007$ components. Correcting our estimated double-peaked $[\mathrm{O}$ III $] \lambda 5007$ fraction of $0.5 \%$ yields a 'true' double $[\mathrm{O}$ III $] \lambda 5007$ fraction of $\sim 2.5 \%$ at $0.8<$ $z<1.6$. The influence of inclination and phase angle for selection of dual AGN through double-peaked emission line profiles was also investigated by Wang \& Zhou (2012) in which they found that, at a phase angle of $\phi \approx 50^{\circ}$, we miss at least $50 \%$ of all AGN with double emission components. We note that this $50 \%$ correction is a lower limit since it does not account for instrumental resolution, and that applying additional corrections based on our completeness estimates would likely make the correction estimated in this manner similar to that of Shen et al. (2011a). Finally, if we take the dual AGN fraction at $z=1.2$ to be $\sim 0.05 \%$ (Yu et al. 2011), then we estimate the fraction of dual AGN out of doublepeaked AGN at $0.8<z<1.6$ to be $2 \%$. This fraction is several times smaller than the results of Fu et al. (2012) (4.5-12\%) and Shen et al. (2011a) ( 10\%) which were obtained from follow-up observations of double-peaked [O III] $\lambda 5007$ AGN. However, the difference can be attributed, at least in part, to the small expected number of dual AGN at $z=1.2$ estimated by Yu et al. (2011) which is due to the redshift evolution of galaxy morphology in their analysis which yields more late-type galaxies with smaller initial SMBH masses at higher redshift.

A direct test of this through follow-up observations is therefore crucial in understanding the frequency of galaxy mergers at redshifts $z>0.8$ and their role in AGN triggering. For example, NIR spectroscopy will be capable of accessing the redshifted [O III $] \lambda 5007$ emission line for sources in our sample, allowing for a direct comparison with the $z<0.8$ samples of double-peaked AGN. This was done with the $z=1.175$ dual AGN candidate CXOXBJ142607.6+353351 in Barrows et al. (2012) which was initially selected through doublepeaked $[\mathrm{Ne} \mathrm{V}] /\left[\begin{array}{ll}\mathrm{Ne} & \mathrm{III}\end{array}\right]$ but for which follow-up NIR spectroscopy provided access to $[\mathrm{O}$ III $] \lambda 5007$. The additional spatial information of $[\mathrm{O}$ III $] \lambda 5007$ provided by $2 \mathrm{D}$ longslit spectroscopy would enable one to determine if any of these sources are strong dual AGN candidates. Follow-up high-resolution imaging, such as radio observations, would be capable of resolving the two AGN cores, if present. 


\section{CONCLUSIONS}

We have compiled a sample of 131 quasars at $z=$ $0.8-1.6$ which show double emission line components in either of the high-ionization narrow lines $[\mathrm{Ne} \mathrm{V}] \lambda 3426$ and $[\mathrm{Ne} \mathrm{III}] \lambda 3869$. The purpose of this search was to identify high-redshift analogs of the doublepeaked [O III $] \lambda 5007$ sources found in several previous studies. Those double-peaked [O III $] \lambda 5007$ sources are believed to represent complex gas kinematics, large-scale outflows, or in a few cases dual AGN. Given the increased frequency of galaxy mergers at higher redshifts and their importance in models of galaxy evolution, we have investigated these phenomena at higher redshifts using our sample, with the following conclusions:

○ There is a clear bias towards selecting double-peaks with large velocity-splittings. This bias was made apparent by our comparison of the velocity-offsets of the blue and red components in our sample to those of [O III]-selected samples, and it is corroborated by the results of our completeness simulations. This selection bias is not surprising, and it is imposed by the relatively weaker intensities of $[\mathrm{Ne} \mathrm{V}] \lambda 3426$ and $[\mathrm{Ne}$ III] $\lambda 3869$ compared to [O III] $] \lambda 5007$.

- We have found two results suggesting that both the blue and red systems are influenced by kinematics in the NLR. First, the line-widths of both the blue and red components are strongly correlated with the line-splittings, suggesting a common origin. Second, we find that the individual offsets for both the 'blue' and 'red' systems are positively correlated with the quasar Eddington ratio, suggesting that the $\mathrm{SMBH}$ accretion rate and therefore the radiation pressure is responsible for driving the line-offsets in the blueward direction for both line components.

○ We find evidence suggesting that the observed kinematics are strongest in the blue systems. This is suggested because the blue systems' have larger velocity shifts from the quasar redshift, those velocity offsets show the strongest correlations with the Eddington ratio, and the blue systems show the highest degree of ionization stratification. This further suggests that the red outflowing components are generally more obscured.

- We have found that a significant fraction $(\sim 23 \%)$ are radio loud, compared to the $10 \%$ radio loud fraction of the parent sample.

Taken together, the previous conclusions paint a picture in which the blue systems originate in a portion of the NLR much closer to the AGN where they are accelerated by the accretion disk radiation pressure or radio jets to high velocities. This explains the large blueshifts, the stronger correlation with Eddington ratio and the pronounced ionization stratification. The red system originates further from the AGN where it is not accelerated to the high velocities of the blue system but is nevertheless close enough so it's bulk velocity offset is also influenced by the AGN radiation pressure. This explains the smaller velocity offsets, the much weaker correlation with Eddington ratio, and the relatively less pronounced ionization stratification compared to the blue systems. A generalized schematic of this scenario in Figure 12. The enhanced radio loud fraction relative to the parent sample also suggests that radio jets may be another mechanism which is capable of accelerating the NLR clouds to produce the line offsets. This sample can be used to study outflows from luminous AGN at relatively high redshifts when AGN feedback may have been an important factor in the growth of massive galaxies.

There are several interesting results which leave open the possibility for dual AGN in the sample. In particular, several of our correlations can be thought of as consistent with the dual AGN scenario, and even suggest that the sample is likely to include dual AGN:

- The correlation between velocity-splitting and Eddington ratio, while consistent with the picture of radiatively-driven outflows, could plausibly be consistent with orbiting SMBH pairs in which enhanced accretion is more likely to occur at smaller separations where the SMBH orbital velocities will be largest.

- We have found that a subset of our sample (40\%) are consistent with no measurable ionization stratification between [ $\mathrm{Ne} \mathrm{V}] \lambda 3426$ and [ $\mathrm{Ne} \mathrm{III]} \lambda 3869$, similar to other dual AGN and strong candidate dual AGN.

- We have found that our sample shows a correlation between the velocity-offset ratio and the luminosity ratio of the blue and red components. This correlation is broadly consistent with the theoretical expectation for a binary, Keplerian orbit, though our sample seems to be systematically offset above the relation. We have shown how this deviation could be produced in a sample which includes a combination of AGN outflows and dual AGN.

- We have estimated the fraction of dual AGN out of double-peaked AGN that we expect at $0.8<z<$ 1.6 , finding a fraction $(2 \%)$ which is smaller than that estimated at lower redshifts. However, we caution that a significant - and perhaps the primary reason for this lower fraction is the small estimated number of high redshift dual AGN that we adopt.

Follow-up NIR observations to access the $\left[\begin{array}{ll}\mathrm{O} & \mathrm{III}\end{array}\right] \mathrm{5007}$ line in our sources would allow for a direct comparison with the $[\mathrm{O}$ III $] \lambda 5007$ velocity and spatial profiles of the $z<0.80$ samples, allowing for a more robust assessment of the origin of the double-peaks in the high-ionization narrow emission lines. Therefore, this sample represents an initial step toward extending the study of double-peaked emission line AGN to higher redshifts.

We would like to thank an anonymous referee for very helpful comments that improved the quality of the paper. We would also like to acknowledge Daniel Stern for assistance on the analysis of our data and 
Laura Blecha for highly useful suggestions regarding the interpretation of our results. Finally, we acknowledge constructive discussion from members of the Arkansas Galaxy Evolution Survey (AGES) and the Arkansas Center for Space and Planetary Sciences, including Douglas Shields, Benjamin Davis, Adam Hughes and
Jennifer Hanley. This research has made use of NASA's Astrophysics Data System and the Sloan Digital Sky Survey. Support for this work was provided in part by the Arkansas NASA EPSCoR program (grant number NNX08AW03A).

\section{REFERENCES}

Abazajian, K. N., et al. 2009, ApJ, 182, 543

Antonucci, R. 1993, ARA\&A, 31, 473

Arribas, S., Mediavilla, E., \& Garcia-Lorenzo, B. 1996, ApJ, 463, 509

Barrows, R. S., et al. 2012, ApJ, 744, 7

Bennert, N., Falcke, H., Schulz, H., Wilson, A. S., \& Wills, B. J. 2002, ApJ, 574, L105

Berrier, J. C., Bullock, J. S., Barton, E. J., Guenther, H. D. Zentner, A. R., \& Wechsler, R. H. 2006, ApJ, 652, 56

Berrier, J. C., \& Cooke, J. 2012, ArXiv e-prints

Bianchi, S., Chiaberge, M., Piconcelli, E., Guainazzi, M., \& Matt, G. 2008, MNRAS, 386, 105

Blecha, L., Loeb, A., \& Narayan, R. 2012, ArXiv e-prints

Cisternas, M., et al. 2011, ApJ, 726, 57

Civano, F., et al. 2010, ApJ, 717, 209

- 2012, ApJ, 752, 49

Comerford, J. M., Gerke, B. F., Stern, D., Cooper, M. C., Weiner, B. J., Newman, J. A., Madsen, K., \& Barrows, R. S. 2012, ApJ 753,42

Comerford, J. M., Griffith, R. L., Gerke, B. F., Cooper, M. C., Newman, J. A., Davis, M., \& Stern, D. 2009a, ApJ, 702, L82

Comerford, J. M., Pooley, D., Gerke, B. F., \& Madejski, G. M. 2011, ApJ, 737, L19+

Comerford, J. M., et al. 2009b, ApJ, 698, 956

Conselice, C. J., Bershady, M. A., Dickinson, M., \& Papovich, C. 2003, AJ, 126,1183

Crenshaw, D. M., Kraemer, S. B., Schmitt, H. R., Jaffé, Y. L.

Deo, R. P., Collins, N. R., \& Fischer, T. C. 2010a, AJ, 139, 871

Crenshaw, D. M., Schmitt, H. R., Kraemer, S. B., Mushotzky, R. F., \& Dunn, J. P. 2010b, ApJ, 708, 419

De Robertis, M. M., \& Osterbrock, D. E. 1984, ApJ, 286, 171

Di Matteo, T., Springel, V., \& Hernquist, L. 2005, Nature, 433, 604

Eracleous, M., \& Halpern, J. P. 2003, ApJ, 599, 886

Everett, J. E. 2007, Ap\&SS, 311, 269

Everett, J. E., \& Murray, N. 2007, ApJ, 656, 93

Ferland, G. J., \& Osterbrock, D. E. 1986, ApJ, 300, 658

Ferrarese, L., \& Merritt, D. 2000, ApJ, 539, L9

Fischer, T. C., Crenshaw, D. M., Kraemer, S. B., Schmitt, H. R., Mushotsky, R. F., \& Dunn, J. P. 2011, ApJ, 727, 71

Fu, H., Myers, A. D., Djorgovski, S. G., \& Yan, L. 2011a, ApJ, 733, 103

Fu, H., Yan, L., Myers, A. D., Stockton, A., Djorgovski, S. G., Aldering, G., \& Rich, J. A. 2012, ApJ, 745, 67

Fu, H., et al. 2011b, ApJ, 740, L44

Ge, J.-Q., Hu, C., Wang, J.-M., Bai, J.-M., \& Zhang, S. 2012, ApJS, 201, 31

Gerke, B. F., et al. 2007, ApJ, 660, L23

Green, P. J., Myers, A. D., Barkhouse, W. A., Mulchaey, J. S., Bennert, V. N., Cox, T. J., \& Aldcroft, T. L. 2010, ApJ, 710, 1578

Guainazzi, M., Piconcelli, E., Jiménez-Bailón, E., \& Matt, G. 2005, A\&A, 429, L9

Heckman, T. M., Miley, G. K., van Breugel, W. J. M., \& Butcher, H. R. 1981, ApJ, 247, 403

Hewett, P. C., \& Wild, V. 2010, MNRAS, 405, 2302

Ho, L. C. 2002, ApJ, 564, 120

Holt, J., Tadhunter, C. N., \& Morganti, R. 2003, MNRAS, 342, 227

- 2008, MNRAS, 387, 639

Hopkins, P. F., Hernquist, L., Cox, T. J., Di Matteo, T., Martini, P., Robertson, B., \& Springel, V. 2005, ApJ, 630, 705

Hudson, D. S., Reiprich, T. H., Clarke, T. E., \& Sarazin, C. L. 2006, A\&A, 453, 433

Kauffmann, G., \& Haehnelt, M. 2000, MNRAS, 311, 576

Kocevski, D. D., et al. 2012, ApJ, 744, 148

Komatsu, E., et al. 2011, ApJS, 192, 18

Komossa, S., Burwitz, V., Hasinger, G., Predehl, P., Kaastra, J. S., \& Ikebe, Y. 2003, ApJ, 582, L15

Komossa, S., Xu, D., Zhou, H., Storchi-Bergmann, T., \& Binette, L. 2008, ApJ, 680, 926

Koss, M., et al. 2011, ApJ, 735, L42+
Kriss, G. 1994, Astronomical Data Analysis Software and Systems, 3, 437

Krolik, J. H. 1999, Active galactic nuclei : from the central black hole to the galactic environment, ed. Krolik, J. H.

Liu, X., Civano, F., Shen, Y., Green, P. J., Greene, J. E., \& Strauss, M. A. 2012a, ArXiv e-prints

Liu, X., Greene, J. E., Shen, Y., \& Strauss, M. A. 2010a, ApJ, 715, L30

Liu, X., Shen, Y., \& Strauss, M. A. 2012b, ApJ, 745, 94

Liu, X., Shen, Y., Strauss, M. A., \& Greene, J. E. 2010b, ApJ, 708,427

Liu, X., Shen, Y., Strauss, M. A., \& Hao, L. 2011, ApJ, 737, 101

Lotz, J. M., Jonsson, P., Cox, T. J., Croton, D., Primack, J. R., Somerville, R. S., \& Stewart, K. 2011, ApJ, 742, 103

Marconi, A., \& Hunt, L. K. 2003, ApJ, 589, L21

Mazzarella, J. M., et al. 2012, ArXiv e-prints

McGurk, R. C., Max, C. E., Rosario, D. J., Shields, G. A., Smith, K. L., \& Wright, S. A. 2011, ApJ, 738, L2

McLure, R. J., \& Jarvis, M. J. 2002, MNRAS, 337, 109

Nelson, C. H., \& Whittle, M. 1996, ApJ, 465, 96

Nesvadba, N. P. H., Lehnert, M. D., De Breuck, C., Gilbert, A. M., \& van Breugel, W. 2008, A\&A, 491, 407

Newman, J. A., et al. 2012, ArXiv e-prints

Osterbrock, D. E., \& Ferland, G. J. 2006, Astrophysics of gaseous nebulae and active galactic nuclei, ed. Osterbrock, D. E. \& Ferland, G. J.

Piconcelli, E., et al. 2010, ArXiv e-prints

Rosario, D. J., McGurk, R. C., Max, C. E., Shields, G. A., Smith, K. L., \& Ammons, S. M. 2011, ApJ, 739, 44

Rosario, D. J., Shields, G. A., Taylor, G. B., Salviander, S., \&

Smith, K. L. 2010, ApJ, 716, 131

Sanders, D. B., Soifer, B. T., Elias, J. H., Madore, B. F. Matthews, K., Neugebauer, G., \& Scoville, N. Z. 1988, ApJ, 325,74

Schneider, D. P., et al. 2010, AJ, 139, 2360

Shen, Y., Liu, X., Greene, J. E., \& Strauss, M. A. 2011a, ApJ, 735,48

Shen, Y., \& Loeb, A. 2010, ApJ, 725, 249

Shen, Y., et al. 2011b, ApJS, 194, 45

Silk, J., \& Rees, M. J. 1998, A\&A, 331, L1

Smith, K. L., Shields, G. A., Bonning, E. W., McMullen, C. C., Rosario, D. J., \& Salviander, S. 2010, ApJ, 716, 866

Spoon, H. W. W., \& Holt, J. 2009, ApJ, 702, L42

Stoughton, C., et al. 2002, AJ, 123, 485

Strateva, I. V., et al. 2003, AJ, 126, 1720

Sturm, E., Lutz, D., Verma, A., Netzer, H., Sternberg, A., Moorwood, A. F. M., Oliva, E., \& Genzel, R. 2002, A\&A, 393, 821

Tadhunter, C. N., Fosbury, R. A. E., di Serego Alighieri, S., Bland, J., Danziger, I. J., Goss, W. M., McAdam, W. B., \& Snijders, M. A. J. 1988, MNRAS, 235, 403

Treister, E., Natarajan, P., Sanders, D. B., Urry, C. M.,

Schawinski, K., \& Kartaltepe, J. 2010, Science, 328, 600

Treister, E., Schawinski, K., Urry, C. M., \& Simmons, B. D. 2012 ApJ, 758, L39

Tsuzuki, Y., Kawara, K., Yoshii, Y., Oyabu, S., Tanabé, T., \&

Matsuoka, Y. 2006, ApJ, 650, 57

Van Wassenhove, S., Volonteri, M., Mayer, L., Dotti, M., Bellovary, J., \& Callegari, S. 2012, ApJ, 748, L7

Vanden Berk, D. E., et al. 2001, AJ, 122, 549

Veilleux, S. 1991, ApJ, 369, 331

Veilleux, S., Shopbell, P. L., \& Miller, S. T. 2001, AJ, 121, 198

Véron-Cetty, M.-P., Joly, M., \& Véron, P. 2004, A\&A, 417, 515

Wang, J., Chen, Y., Hu, C., Mao, W., Zhang, S., \& Bian, W. 2009, ApJ, 705, L76

Wang, X.-W., \& Zhou, H.-Y. 2012, ApJ, 757, 124

Whittle, M. 1992, ApJ, 387, 109

Xu, D., \& Komossa, S. 2009, ApJ, 705, L20

Yu, Q., Lu, Y., Mohayaee, R., \& Colin, J. 2011, ApJ, 738, 92

Zamanov, R., Marziani, P., Sulentic, J. W., Calvani, M.

Dultzin-Hacyan, D., \& Bachev, R. 2002, ApJ, 576, L9

Zheng, W., Sulentic, J. W., \& Binette, L. 1990, ApJ, 365, 115 